\title{
Age Patterns in Risk Taking Across the World
}

\author{
Natasha Duell ${ }^{1}$ Laurence Steinberg ${ }^{2} \cdot$ Grace Icenogle $^{1} \cdot$ Jason Chein $^{1}$ • \\ Nandita Chaudhary ${ }^{3}$. Laura Di Giunta ${ }^{4}$ Kenneth A. Dodge ${ }^{5} \cdot$ Kostas A. Fanti $^{6}$. \\ Jennifer E. Lansford ${ }^{5}$ - Paul Oburu ${ }^{7}$. Concetta Pastorelli ${ }^{4}$ - Ann T. Skinner ${ }^{5}$. \\ Emma Sorbring $^{8} \cdot$ Sombat Tapanya $^{9}$ - Liliana Maria Uribe Tirado ${ }^{10}$. \\ Liane Peña Alampay ${ }^{11}$ - Suha M. Al-Hassan ${ }^{12}$ - Hanan M. S. Takash ${ }^{13}$. \\ Dario Bacchini $^{14} \cdot$ Lei Chang ${ }^{15}$
}

Received: 11 July 2017 / Accepted: 14 September 2017

(C) Springer Science+Business Media, LLC 2017

\begin{abstract}
Epidemiological data indicate that risk behaviors are among the leading causes of adolescent morbidity and mortality worldwide. Consistent with this, laboratorybased studies of age differences in risk behavior allude to a peak in adolescence, suggesting that adolescents demonstrate a heightened propensity, or inherent inclination, to take risks. Unlike epidemiological reports, studies of risk taking propensity have been limited to Western samples, leaving questions about the extent to which heightened risk taking propensity is an inherent or culturally constructed aspect of adolescence. In the present study, age patterns in risk-taking propensity (using two laboratory tasks: the Stoplight and the BART) and real-world risk taking (using self-reports of health and antisocial risk taking) were examined in a sample of 5227 individuals (50.7\% female) ages $10-30(M=17.05$ years, $\mathrm{SD}=5.91)$ from 11 Western
\end{abstract}

Natasha Duell

ntduell@temple.edu

1 Department of Psychology, Temple University, Philadelphia, PA, USA

2 Department of Psychology, Temple University and King Abdulaziz University, Philadelphia, PA, USA

3 Department of Human Development and Childhood Studies, Lady Irwin College, University of Delhi, New Delhi, India

4 Department of Psychology, Università di Roma "La Sapienza", Roma, RM, Italy

5 Center for Child and Family Policy, Duke University, Durham, NC, USA

6 Department of Psychology, University of Cyprus, Kallipoleos, Cyprus

7 Department of Educational Psychology, Maseno University, Maseno, Kenya and non-Western countries (China, Colombia, Cyprus, India, Italy, Jordan, Kenya, the Philippines, Sweden, Thailand, and the US). Two hypotheses were tested: (1) risk taking follows an inverted-U pattern across age groups, peaking earlier on measures of risk taking propensity than on measures of real-world risk taking, and (2) age patterns in risk taking propensity are more consistent across countries than age patterns in real-world risk taking. Overall, risk taking followed the hypothesized inverted-U pattern across age groups, with health risk taking evincing the latest peak. Age patterns in risk taking propensity were more consistent across countries than age patterns in real-world risk taking. Results suggest that although the association between age and risk taking is sensitive to measurement and culture, around the world, risk taking is generally highest among late adolescents.
8 Department of Psychology, University West, Trollhättan, Sweden

9 Department of Psychiatry, Chiang Mai University, Chiang Mai, Thailand

10 Consultorio Psicológico Popular, Universidad San Buenaventura, Medellín, Colombia

11 Department of Psychology, Ateneo de Manila University, Metro Manila, Philippines

12 Hashemite University and Emirates College for Advanced Education, Al Zafranah, Abu Dhabi, United Arab Emirates

13 Queen Rania Faculty for Childhood, Hashemite University, Zarqa, Jordan

14 Department of Psychology, University of Campania "Luigi Vanvitelli", Caserta, CE, Italy

15 Department of Psychology, University of Macau, Zhuhai Shi, China 
Keywords Adolescents $\cdot$ Risk taking $\cdot$ Development • Cross-national

\section{Introduction}

Cross-national comparisons of adolescent risk taking indicate great variability in the prevalence of various risk behaviors around the world (World Health Organization [WHO] 2014). For example, rates of weekly cigarette smoking among 15-year-olds are as low as $8 \%$ in the United States, Canada, and Norway, but as high as $56 \%$ in Romania and Denmark. Over $50 \%$ of sexually active teens in sub-Saharan Africa report not having used a condom the last time they had intercourse, whereas in Germany and France, this rate is below 20\%. Rates of risk taking also vary within countries as a function of the specific behavior involved. Whereas rates of cannabis use among 15-yearolds are at 30\% in the United States and Spain, more than twice as many Spanish than American teens have been drunk at least twice in their lifetime ( $34 \%$ vs. $14 \%$, respectively).

Different rates of risk behavior across countries are likely attributable to the fact that youth growing up in different parts of the world are exposed to different societal norms and have different opportunities to engage in risky behaviors (Steinberg 2014). In many Asian countries, for instance, behavior is regulated via strict societal norms (Hofstede 2011), such as the expectation that youth will spend long hours in academic and structured extracurricular activities (e.g., Stevenson and Zusho 2002), which in turn may limit opportunities to engage in risky behavior (Jessor et al. 2003). Further, some countries forbid specific behaviors for religious reasons. Drinking alcohol, for example, is prohibited in some Muslim countries, markedly reducing the prevalence of drinking (Mauseth et al. 2016). Public health practices are also important influences on risk behaviors; in some African countries that have exceptionally high rates of HIV/AIDS, access to condoms may be a stronger predictor of sexual risk taking (Hendriksen et al. 2007) than psychological factors such as impulsivity (Maticka-Tyndale and Tenkorang 2010).

Despite such variations in the types and rates of risk behaviors reported by adolescents across the world, two commonalities are noteworthy. First, in most parts of the world, especially in the developed world, the leading causes of morbidity and mortality among youth are unintentional accidents resulting from risky behaviors, violence, and drug use (WHO 2014). Researchers from many countries, including Brazil (Anteghini et al. 2001), China (Xing et al. 2006), Japan (Takakura et al. 2001), and the United States
(Willoughby et al. 2013) have cited adolescent risk taking (e.g., drug use, unprotected sex, cigarette smoking, binge drinking, and fighting) as a major public health concern. The second noteworthy commonality in risk behavior is that in most parts of the world, risky behavior increases over the course of adolescence before declining as individuals reach their 20s (e.g., Eisner 2002), following the "age-crime curve" that has been observed consistently across time and cultures (Hirschi and Gottfredson 1983; Piquero et al. 2003). Regarding the increase during adolescence, for example, a study of Japanese students conducted by Takakura and colleagues (2001) found that risk behaviors such as cigarette smoking, drinking alcohol, and having intercourse were greater among older (18-year old) than younger (15-year-old) adolescents. A study of alcohol use among Italian youth demonstrated greater drinking among young adults ages 18-19 compared to teens ages 14-15 (Donato et al. 1995). Furthermore, a study of Romanian youth ages 11-15, 15-19, and 19-25 (Lotrean et al. 2010) indicated that fighting, vandalism, smoking, and intoxication was greater in each successive age group (although for some risks, such as illicit drug use, teens ages 15-19 did not differ from young adults). Consistent with epidemiological reports (WHO 2014), risk behavior in each of these studies was greater among males compared to females in all age groups.

Among the few international studies with both adolescent and adult samples, findings have alluded to a gradual decline in risk taking that begins sometime between late adolescence and the mid-20s, among both males and females (Fuller et al. 2015; Plant et al. 2009; Willoughby et al. 2013). Across several countries, both violent and property crime decline during the early 20 s, falling off markedly after 25 years (cf., Eisner 2002). Binge drinking (Plant et al. 2009; Willoughby et al. 2013), cannabis use (Eisner 2002), and sexual promiscuity (Fuller et al. 2015) in many countries also begin to decrease before the mid-20s. There are of course some exceptions. For example, rates of cigarette smoking (Anderson Johnson et al. 2006) and binge drinking (Plant et al. 2009) in several countries such as China (cigarette smoking), and the UK and Spain (binge drinking), continue increasing into adulthood, past age 25 . Despite these international differences in absolute rates of adolescent risk behavior, there is evidence to suggest that, on average, an increase in risk taking during adolescence and a decline during young adulthood is seen virtually around the world, across countries that differ markedly in cultural norms and opportunities for risk taking.

A growing literature on adolescent brain development may help explain why this is the case. Findings from neuroscientific studies have suggested that brain regions responsible for reward processing mature earlier than those necessary for cognitive control (Casey et al. 2011). One consequence of this is that the sensitivity of the reward system is heightened 
during the second decade of life, making youth more sensitive to the potential rewards of various risks and challenging youths' self-regulatory capabilities (Casey et al. 2011). Dual systems theorists posit that due to this maturational imbalance, adolescence is the developmental period during which individuals possess the greatest propensity, or inherent inclination, to take risks (Steinberg 2008; see also Spear 2013). From this perspective, it is plausible that cross-national similarities in patterns of risk taking stem from a biological propensity toward risk taking that is greatest in adolescence and comparatively weaker in adulthood, despite variations among countries in the specific types of risks in which youth engage. In other words, adolescence may be a time at which individuals are more inclined to take risks, even though the specific risks they take vary by the opportunities and norms of their culture.

In epidemiological studies, risk taking is generally defined as engaging in behaviors with the potential to compromise the health and well-being of the individual and those around him or her (Hawley 2011). However, defining risk in this way can be problematic when attempting to compare patterns of risk taking across cultures due to cultural variability in the types of behaviors that are considered bad, harmful, or socially unacceptable. For example, dating in Western societies is generally viewed as a normative behavior that allows teenagers to develop healthy attachments to their peers. In contrast, in many Islamic societies, where premarital dating is culturally (and in some cases legally) prohibited, associations between unmarried males and females may be risks that threaten the reputation of both the youth and their families (Rahbari 2016).

One way of addressing this issue is to examine risktaking propensity, or the inherent inclination to take risks. Measuring risk-taking propensity can be achieved using laboratory-based behavioral risk-taking tasks that minimize contextual confounds related to differences in societal and cultural norms and opportunities for risk. On these tasks, risk taking is defined as choosing a course of action for which the likelihood of a given outcome is uncertain. This broad conceptualization of risk casts a wider net of possible behaviors in which youth can engage, and assessing risk propensity through behavioral tasks is thought to capture individuals' underlying inclinations for risk rather than realworld behavior that is affected by contextual factors. Although it is important to know the prevalence of actual risk behavior in different parts of the world, measures of risk-taking propensity are better-suited to address the question of whether adolescents in general demonstrate a heightened propensity for risk taking than adults. Together, comparisons of both real-world risk taking and risk-taking propensity offer a unique comparison of the extent to which age patterns in risk-taking propensity match age differences in real-world risk behavior.
The distinction between risk-taking propensity and realworld risk taking is frequently overlooked (see, e.g., Willoughby et al. 2013), but is particularly important for both cross-sectional and cross-national research. Unlike risktaking propensity, which is measured with lab-based tasks that minimize contextual confounds, real-world risk taking, assessed via self-report surveys, is influenced by cultural expectations for adolescent behavior as well as the freedoms or constraints that provide chances for, or place limits on, adolescents' opportunities to engage in risk behaviors. Although adolescents from different cultures may evince a biological propensity for risk taking, this propensity interacts with cultural and environmental forces that shape its expression (Choudhury 2010; Spear 2013). Accordingly, whereas studies of risk-taking propensity have suggested that risk taking peaks in adolescence, studies of selfreported risk taking have demonstrated that some forms of health risk taking, such as binge drinking, peak in early adulthood (e.g., Eisner 2002; Willoughby et al. 2013). This is not surprising, given that older individuals typically have more opportunities than adolescents to engage in many health risk behaviors (e.g., in the US, young adults can purchase alcohol and cigarettes legally). This may also be true, although to a lesser extent, for antisocial risk taking. For example, it is easier for older adolescents to engage in delinquent or antisocial behaviors such as vandalism because they are less closely monitored by adults (Osgood and Anderson 2004). On the other hand, as a minor, it may be easier to vandalize property or get into a fight than to get access to cigarettes or alcohol. To the extent that this is true, we would expect age patterns in antisocial risk taking to be similar to age patterns in risk-taking propensity (i.e., evincing an inverted U-shape pattern with greater risk taking among adolescents), but age patterns in health risk taking to evince relatively greater risk behavior among young adults. Thus, examining risk-taking propensity minimizes the effects of both cultural variability across countries and contextual factors (such as age-related opportunities for risk taking) that operate within individual cultures.

Although several researchers have examined age patterns in risk-taking propensity in Western cultures (see Shulman et al. 2016 for a review), few researchers have explored risk-taking propensity in non-Western samples. Further, no studies to our knowledge have compared age patterns in both real-world risk taking and risk-taking propensity within the same sample. However, two recent cross-cultural studies of sensation seeking and impulsivity, constructs thought to represent the psychological manifestations of reward processing and cognitive control, respectively (Smith et al. 2013), are informative. In one, Steinberg and colleagues (2017) found notable similarities across eleven countries in Africa, Asia, Europe, the Middle East, and the Americas in the development of sensation seeking and 
impulse control. Consistent with previous work in Western samples (see Shulman et al. 2016), Steinberg and colleagues (2017) found that in a majority of the countries studied, sensation seeking peaked in adolescence, whereas impulse control continued to develop into early adulthood. In another analysis of the same data, Duell and colleagues (2016) found that sensation seeking was associated with risk taking (using a composite of behavioral and self-report measures) in both Asian and Western countries (although poor self-regulation was associated with risk taking only in Western nations). Such findings suggest that the processes thought to underlie risk taking may be similar across countries, which may account for cross-national similarities in relatively heightened risk taking propensity during adolescence. To the extent that certain aspects of neurobiological development are inherent to adolescence (Spear 2013), the inclination to take risks may be heightened during the second decade of life regardless of culture.

\section{Current Study}

Epidemiological research on risk taking suggests that adolescent risk behavior is a public health concern in many parts of the world. Neurodevelopmental studies and studies employing lab-based tasks of risk taking suggest that adolescents possess a heightened propensity for risk behavior compared to adults. However, most studies of risk-taking propensity have been conducted in Western samples, leaving unclear the extent to which heightened risk propensity in adolescence is culturally variable or culturally consistent. In the present study, we compared age patterns in both risktaking propensity and real-world risk taking in Western and non-Western samples to examine (a) whether the age patterns of each were similar and (b) the extent to which the age patterns were similar or different across countries. We hypothesized that across countries, risk taking would be comparatively higher among late adolescents than younger adolescents or adults, and that age patterns in risk-taking propensity would be more homogeneous across countries than age patterns in real-world risk taking. This study adds to the literature a deeper understanding of cross-cultural similarities and differences in the presence and timing of an adolescent-specific peak in risk taking both with respect to an underlying propensity to engage in risky behavior in labbased experiments as well as to risk taking in the real world.

\section{Methods}

\section{Participants}

The sample for the present analyses included 5227 individuals ages $10-30(M=17.05$ years; $\mathrm{SD}=5.91)$ from primarily metropolitan areas within 11 countries: GuangZhou and Shanghai, China $(n=489)$; Medellin, Colombia $(n=498)$; Nicosia, Cyprus $(n=364)$; Delhi, India $(n=$ 417); Naples and Rome, Italy ( $n=547)$; Amman and Zarqa, Jordan $(n=450)$; Kisumu, Kenya $(n=483)$; Manila, the Philippines $(n=505)$; several cities in the west of Sweden $(n=416)$; Chiang Mai, Thailand $(n=502)$; and Durham and Winston-Salem, NC, the United States $(n=556)$. The gender balance was nearly even within the full sample (50.7\% female, $n=2652$ ), as well as within each country (range: $46.7-53.3 \%$ female). Most of the 10-11-year-olds were participants in an ongoing study of parenting across cultures, Parent Behavior and Child Adjustment Across Cultures (PAC; Lansford and Bornstein 2011) that is being conducted in all of these locales except Cyprus and India. This study was approved by the participating university's Institutional Review Board (IRB; approval 2032).

The PAC countries were originally selected because they differ markedly in how children are disciplined, a primary focus of that project. This focus resulted in a sample of countries that was diverse along several socio-demographic dimensions, including predominant race/ethnicity, predominant religion, various economic indicators, and indices of child well-being. For example, on the Human Development Index, a composite measure of a country's status with respect to health, education, and income, participating countries ranged from a rank of 5 (US) to 147 (Kenya) out of 187 countries with available data (United Nations Development Programme 2014). The participating countries also varied widely on psychological constructs such as individualism vs. collectivism, which likely influence how adolescents and adults make day-to-day decisions. Ultimately, this diversity provided us with an opportunity to examine our research questions in a sample that was more generalizable to a wider range of the world's population than is typical in most research on adolescence.

All participants were recruited from the same neighborhoods as the children in the PAC study. In Cyprus and India, which are not in the PAC study, we recruited from neighborhoods similar to those used in the PAC study. Many contemporary scholars define adolescence as beginning with puberty and ending when individuals have made the transition into adult roles. The 10-30 age range in this study allowed us to capture this age period while allowing for worldwide variation in the age of pubertal onset and the age of transition to adulthood. Across countries, participants came from households that were primarily working and middle class, based on parental education (the average was "some college") and that had similar standings in terms of within-country socioeconomic status. Participants in all but the US did not identify as being members of any ethnic minority groups. In the US, we aimed to enroll approximately equal numbers of Black, Latino, and White 
participants so that the sample was ethnically diverse (although not necessarily representative of the population). Efforts were made to ensure that participants from each country were representative of the cities from which they were recruited. However, given that various regions within the individual countries vary with respect to geography, ethnic origins, and cultural practices, our country-specific samples cannot be assumed to be representative of the country's entire population.

\section{Procedures}

Participants were recruited via flyers posted in neighborhoods and schools, ads placed in newspapers, and word of mouth. Because of the varied recruitment methods, we could not determine whether those who responded to recruitment ads differed from those who did not. In keeping with the IRB requirements in each country, informed consent was obtained from all adults age 18 and older, and parental consent and adolescent assent were acquired for all individuals younger than $18^{1}$. Local IRBs approved all procedures.

Research staff in all countries underwent identical training procedures. Participants completed a 2-h test battery administered by a trained research assistant on a laptop computer (purchased by the participating university for the purposes of this study) that included behavioral tasks, selfreport measures, a demographic questionnaire, and an intelligence assessment. These assessments were completed individually in participants' homes, schools, or other locations designated by the participants, with the approval of the research assistant.

To keep participants engaged, they were told they would receive a base payment for participating, and that they could obtain a bonus based on their performance on the computer tasks. In actuality, all participants received the bonus. This strategy was used to increase motivation to perform well on tasks but ensure that no participants were penalized for their performance. In the United States, the base payment was US\$30 and the bonus was US\$15. In other countries, the principal investigators and site coordinators (with the approval of local IRBs) determined an appropriate amount of payment, accounting for the local standard of living and minimum wage, and ensuring that the amount was sufficient to encourage participation but not so large so as to be coercive. (The participating university in Sweden did not permit research subjects to be paid in cash, so participants were given three movie tickets [two as the base payment and one as a bonus] as compensation.)

\footnotetext{
${ }^{1}$ In Sweden, informed consent was obtained from all participants age 15 and older, and parental consent and adolescent assent were acquired for individuals younger than 15 , per Swedish law.
}

Following each assessment, the interviewer answered five questions about the participant's engagement in the assessment and the quality of the data. A small number of cases $(3.2 \%, N=172)$ were rated as unusable (e.g., the participant did not appear to understand the questions or tasks, did not pay attention to instructions, or was obviously disengaged); these cases were dropped from the analyses.

\section{Measures}

Measures were administered in the predominant language at each site, following forward- and back-translation and meetings to resolve ambiguities in linguistic or semantic content (Erkut 2010; Maxwell 1996). Translators were research assistants fluent in English and the target language. In addition to translating the measures, translators noted items that did not translate well, were inappropriate for the participants, were culturally insensitive, or elicited multiple meanings, and worked with site coordinators to make appropriate modifications. Each country used the same version of the measures, which were approved by translators at all sites. Measures were administered in Mandarin Chinese (China), Spanish (Colombia and the United States), Italian (Italy), Arabic (Jordan), Dholuo (Kenya), Filipino (the Philippines), Greek (Cyprus), Hindi (India), Swedish (Sweden), Thai (Thailand), and American English (India, Kenya, the Philippines, and the United States).

\section{Stoplight}

The Stoplight game (Steinberg et al. 2008) is a computerized behavioral measure of risk-taking propensity on which performance has been linked to real-world risk taking among adolescents (Kim-Spoon et al. 2016). Participants were asked to "drive" a car to a radio station for a prize in as little time as possible, and had to pass through 20 intersections, each marked by a traffic signal. The participant's vantage point was that of someone behind the wheel. Before playing, participants were informed that when approaching an intersection in which the traffic signal turned yellow, they had to decide whether to stop the car (using the space bar) and wait for the light to cycle back to green, or attempt to cross the intersection. Participants could not control the car's speed, and the brake only worked after the light turned yellow. Participants were told that one of three things could happen depending on their decision: (a) if brakes were not applied and the car passed through the intersection without crashing, no time was lost, (b) if brakes were applied before the light turned red, the car would stop safely, but $3 \mathrm{~s}$ would be lost waiting for the green light, or (c) if brakes were not applied or applied too late and the car crashed (accompanied by squealing tires, a loud crash, and the image of a shattered windshield), $6 \mathrm{~s}$ would be lost. Participants had to 
decide whether to drive through the intersection to save time (but risk losing time if a crash occurred), or to stop and wait (and willingly lose a smaller amount of time).

Among the 20 intersections, there was one in which the light remained green and all cars passed through (data from this intersection were not used). There were 14 intersections in which the latency between the yellow and red lights was long enough for participants to stop; 10 of these were configured so that running the red light resulted in a crash. For two additional intersections, the latency between the yellow light and crossing vehicle was so short that almost all participants crashed. Finally, for three intersections, the latency between the yellow light and crossing vehicle was long enough that participants could run the red light without crashing. Risk was measured as the percentage of intersections participants entered without braking (crashes + thrus / intersections).

\section{Balloon analogue risk task (BART)}

A modified version of the BART (adapted from Lejuez et al. 2002) developed for use in brain imaging studies was implemented. This computerized task of risk-taking propensity has demonstrated strong validity in previous studies (Defoe et al. 2015; Lejuez et al. 2003). The task included 20 trials in which participants decided how much air to "pump" into a balloon; the larger the balloon inflated, the more points were earned. Pressing the space bar initiated inflation. The balloon inflated continuously until the participant paused inflation by pressing the space bar again. From this point, participants could incrementally inflate the balloon by pressing the space bar. When the desired inflation size was reached, participants hit a separate key to obtain the points accumulated. At some point, the addition of more air caused the balloon to burst, in which all points earned during that trial were lost. Each balloon had a unique maximum inflation point that was predetermined on each trial but unknown by the participant; the more the participant allowed the balloon to inflate, the greater risk he or she incurred. Risk taking was operationalized as the average inflation percentage across the 20 trials [(inflated size of a balloon/maximum inflation point $) \times 100]$, with higher inflation percentages indicating greater risk taking.

\section{Self-reported risk taking}

Self-reported risk taking was measured on a subscale of the Benthin Risk Perception scale (Benthin et al. 1993). The measure included a list of nine risky activities and asked participants whether they had (coded 1$)$ or had not (coded 0$)$ engaged in the activity within the past 6 months. Participants also reported on the frequency, or how often they engaged in each activity, on a scale of 1 (None) to 4 (More than 5 times). Because opportunities to engage in various health-risk behaviors are more likely to be constrained by age than opportunities to engage in various forms of antisocial risk taking, we calculated two separate variety scores for the risk items: (1) the percent of all health-related risks (drinking alcohol, getting in the car with a drunk driver, smoking cigarettes, and having unprotected $\mathrm{sex}^{2}$ ) endorsed; and (2) the percent of all antisocial risks (vandalizing, stealing, fighting, walking through a dangerous neighborhood, and threatening someone) endorsed. The percentage of individuals engaging in each of these risk behaviors, broken down by age and country, are available from the authors upon request.

Only the variety scores were used for the present analyses. Variety scores are widely used in criminological research because they are highly correlated with frequency measures but less susceptible to participant recall bias and unreliable estimates, a problem in the case of activities that occur frequently among some individuals (e.g., fighting). Thus, while variety and frequency scores are thought to represent the same propensity for risk taking, variety scores are the preferred method of measurement (e.g., Hindelang et al. 1981). To confirm that the variety and frequency scores yielded similar measures of risk taking, we correlated each person's frequency score with his or her variety score. In the present sample, this correlation was very high $(r=.927, p<.001$ for health risks and $r=.893, p<.001$ for antisocial risks). These correlations were comparable across countries.

\section{Covariates}

Two variables that have been linked to adolescent risk behavior are intelligence (Ellis and Walsh 2003) and socioeconomic status (Kipping et al. 2015). In light of this, and because prior analyses of these data indicated small but statistically significant age differences in intellectual ability and socioeconomic status, these variables were used as covariates in all analyses.

\section{Intellectual ability}

The Matrix Reasoning subtest of the Wechsler Abbreviated Scale of Intelligence (WASI) (Psychological Corporation 1999), administered on a laptop, was used to produce an estimate of nonverbal intellectual ability. Other subtests, which rely on verbal ability, were not used due to the variability in language across sites. The WASI has been normed for individuals ages 6-89 years. An age-normed

\footnotetext{
2 This item was omitted for participants under age 13 and all participants from Kenya, Jordan, and India, as requested by the lead researchers at these sites
} 
$T$-score was computed for each participant, with $T$-scores ranging from $20-80$ in the present sample.

\section{Parental education}

Participants provided information on parental education, which was used to index socioeconomic status. Responses were given numeric values that represented completed years of education. A value of 0 indicated no education, values 1 -12 corresponded to grade level (e.g., a value of 10 indicated completion of 10th grade, 13 indicated some college, 14 indicated a college degree, and 15 represented education beyond college). The parent or caregiver's highest level of education was used in single-parent homes and the average of the participant's mother's and father's (or primary caregivers') education levels was used in homes with two parents.

\section{Data Analysis}

All analyses were conducted using Mplus Version 7. Bootstrapping was implemented to account for the positive skew of the self-reported risk-taking scores (Efron and Tibshirani 1993). The full model for all analyses included age, age $^{2}$, gender, and the interactions between gender and both age and age $^{2}$, with country, intellectual ability, and parental education included as covariates. Country was entered as a series of dichotomous variables, with the US omitted as the reference group. This approach was chosen over a multilevel model given that the number of clusters (i.e., countries) was lower than the recommended minimum of 20 (B. Muthen, personal communication, July 6, 2017). All independent variables were centered. Intellectual ability scores and parental education were centered at the grand mean for the full sample and age was centered at 10 years (the youngest age).

We first tested our model across the entire sample. Using regression analyses, we examined age patterns in risk taking for each of the risk measures (Stoplight, BART, selfreported health risk behavior, and self-reported antisocial risk behavior) separately. In the first analysis, country, intellectual ability, parental education, gender, and age were included as independent variables. Then, age ${ }^{2}$ was added to the model. The linear and quadratic terms for age were included in separate steps since, in the context of a significant quadratic effect, the linear effect of age is interpreted as the instantaneous rate of change in risk taking. Thus, examining the linear term in the absence of the quadratic term revealed whether there was a true linear effect of age. Finally, the two interaction terms between age (or age ${ }^{2}$ ) and gender were added to the model.
Next, given our interest in determining whether age patterns in risk taking were similar across countries, we conducted a multi-group analysis, identifying country as a grouping variable. We tested two models for each risk measure separately: (1) constraining the regression paths for age, age ${ }^{2}$, (age $\times$ gender), and $\left(\right.$ age $^{2} \times$ gender) to be equal across countries, and (2) allowing the same regression paths to be estimated freely across countries (while constraining the regression paths for intellectual ability and parental education to be equal across countries in both models). Because we were primarily interested in examining whether the age patterns varied across countries, we ran a second iteration of this analysis in which we excluded the interaction terms between gender and age (or age ${ }^{2}$ ). The results of both sets of analyses were compared. Allowing only the age variables (but not the covariates) to vary freely allowed to us to attribute changes in model fit to the age variables; if the covariates were also free to vary, we would not be able to determine whether the change in model fit was due to age or one of the covariates. Finally, we compared model fit by testing whether the difference in the Chi-Square value between the free and fixed models was significantly different from zero. If the free model was a better fit (indicated by a smaller Chi-Square value), we concluded our model was not equal across countries and tested the model separately in each country.

In order to test our second hypothesis that age patterns in risk-taking propensity would be more consistent across countries than age patterns in real-world risk taking, we ran additional multiple group models including all of the risk taking variables within the same model (we decided to take this approach after determining that including all of the risk variables within the same model did not substantially change the results of our original analyses). So that all of the risk taking measures would be on the same scale, they were standardized using the grand mean and standard deviations for the full sample. We reduced our model to include only parental education, intellectual ability, gender, age and age ${ }^{2}$ as predictors of risk taking. We then compared model fit statistics for four separate models: (1) all regression paths free to vary across countries; (2) all regression paths constrained to be equal across countries; (3) only regression paths for the risk-taking propensity variables (Stoplight and BART) constrained to be equal (while the regression paths for real-world risk taking were free to vary across countries); (4) only regression paths for real-world risk taking variables (health and antisocial) constrained to be equal (while the regression paths for risk-taking propensity were free to vary across countries). If the fit statistics for Model 3 were better than those for Model 4, this indicated more cross-national variability in self-reported risk taking than in risk-taking propensity. 
Table 1 Correlations among main study variables across the entire sample

\begin{tabular}{|c|c|c|c|c|c|c|c|c|}
\hline & 1 & 2 & 3 & 4 & 5 & 6 & 7 & 8 \\
\hline 1. Par Ed. & - & 0.203 & 0.024 & -0.066 & 0.004 & 0.04 & -0.025 & 0.041 \\
\hline 2. Intel. ability & & - & 0.072 & 0.147 & 0.059 & 0.095 & 0.078 & -0.082 \\
\hline 3. Gender & & & - & -0.001 & 0.06 & 0.137 & 0.108 & 0.21 \\
\hline 4. Age & & & & - & $-\mathbf{0 . 0 3 3}$ & 0.184 & 0.529 & -0.025 \\
\hline 5. Stoplight & & & & & - & 0.1 & 0.048 & 0.02 \\
\hline 6. BART & & & & & & - & 0.139 & 0.055 \\
\hline 7. Health risk & & & & & & & - & 0.263 \\
\hline 8. Antisocial risk & & & & & & & - & \\
\hline
\end{tabular}

Gender is dichotomous: $0=$ Female and $1=$ Male. Stoplight and BART are behavioral risk taking propensity tasks, health (drinking alcohol, getting in the car with a drunk driver, smoking cigarettes, and having unprotected sex) and antisocial (vandalizing, stealing, fighting, walking through a dangerous neighborhood, and threatening someone) risks are self-report scales. Bolded correlation coefficients are significant at $p<.05$

Par. Ed. parental education, Intel. ability intellectual ability
Table 2 Unadjusted means and standard deviations for main study variables across countries and within the full sample

\begin{tabular}{|c|c|c|c|c|c|c|c|c|c|c|c|c|}
\hline \multirow[b]{2}{*}{ Country } & \multicolumn{2}{|c|}{ Stoplight } & \multicolumn{2}{|c|}{ BART } & \multicolumn{2}{|c|}{ Health risks } & \multicolumn{2}{|c|}{ Antisocial risks } & \multicolumn{2}{|c|}{ Par. Ed. } & \multicolumn{2}{|c|}{ Intel. ability } \\
\hline & Mean & SD & Mean & SD & Mean & SD & Mean & SD & Mean & SD & Mean & SD \\
\hline China & 45.56 & 21.51 & 74.98 & 9.94 & 21.22 & 24.66 & 9.73 & 15.79 & 10.21 & 2.42 & 52.50 & 8.73 \\
\hline Italy & 44.58 & 17.43 & 69.39 & 10.40 & 28.88 & 32.52 & 22.30 & 21.65 & 11.53 & 2.45 & 9.34 & 9.31 \\
\hline Kenya & 37.08 & 25.82 & 67.46 & 12.99 & 20.55 & 22.57 & 28.65 & 27.49 & 12.23 & 2.10 & 41.03 & 11.91 \\
\hline Philippines & 38.39 & 21.37 & 70.43 & 10.11 & 23.22 & 26.15 & 28.12 & 24.59 & 13.58 & 1.19 & 54.24 & 7.99 \\
\hline Thailand & 45.29 & 20.95 & 69.63 & 10.37 & 27.29 & 30.13 & 23.67 & 22.84 & 9.10 & 3.81 & 46.50 & 11.21 \\
\hline Sweden & 45.95 & 18.75 & 76.75 & 8.85 & 32.03 & 30.80 & 15.82 & 19.36 & 14.46 & 1.18 & 51.07 & 9.55 \\
\hline US & 42.08 & 18.97 & 67.33 & 12.02 & 21.76 & 28.25 & 19.03 & 22.17 & 13.05 & 2.26 & 51.69 & 9.41 \\
\hline Colombia & 43.43 & 18.66 & 70.91 & 9.84 & 28.01 & 28.83 & 26.31 & 23.45 & 10.27 & 3.38 & 44.39 & 11.68 \\
\hline Jordan & 24.79 & 21.03 & 75.99 & 13.81 & 14.67 & 20.74 & 25.07 & 29.77 & 12.13 & 2.64 & 44.44 & 11.77 \\
\hline India & 53.21 & 27.26 & 71.63 & 11.93 & 16.19 & 24.85 & 20.53 & 24.47 & 12.10 & 3.09 & 47.26 & 11.85 \\
\hline Cyprus & 42.21 & 22.32 & 69.91 & 9.97 & 35.03 & 32.02 & 15.44 & 23.00 & 12.19 & 2.01 & 51.62 & 9.08 \\
\hline Full Sample & 42.01 & 22.23 & 71.15 & 11.44 & 24.30 & 28.23 & 21.54 & 24.01 & 11.85 & 2.97 & 48.56 & 11.03 \\
\hline
\end{tabular}

Stoplight and BART are behavioral measures of risk taking propensity; health (drinking alcohol, getting in the car with a drunk driver, smoking cigarettes, and having unprotected sex) and antisocial (vandalizing, stealing, fighting, walking through a dangerous neighborhood, and threatening someone) risks are self-report scales. Possible range of values for all risk items is $0-100$

Par. Ed. parental education, Intel. ability intellectual ability

\section{Results}

The present study examined age patterns in risk-taking propensity and real-world risk taking across 11 Western and non-Western countries. Two hypotheses were tested: (1) risk taking would follow an inverted U-pattern across age groups, peaking earlier on measures of risk-taking propensity than on measures of real-world risk taking, and (2) age patterns in risk-taking propensity would be more consistent across countries than age patterns in real-world risk taking. In the following section, we first report results from the full-sample analyses testing Hypothesis 1 for each of the four risk measures. Then, we report results from the country-specific analyses testing Hypothesis 1 for each of the four risk measures. Finally, we report results from analyses testing Hypothesis 2. Results examining sex differences in age patterns are also reported. Correlations among all variables are presented in Table 1. Means and standard deviations are presented by country in Table 2, and by age in Table 3 . Within-country correlations and bootstrapped confidence intervals for the regression results are available from the authors upon request. 
Table 3 Unadjusted means and standard deviations for risk taking across gender and age groups

\begin{tabular}{|c|c|c|c|c|c|c|c|c|c|}
\hline \multirow{2}{*}{ Age group } & \multirow[b]{2}{*}{ Gender } & \multicolumn{2}{|c|}{ Stoplight } & \multicolumn{2}{|l|}{ BART } & \multicolumn{2}{|c|}{ Health risks } & \multicolumn{2}{|c|}{ Antisocial risks } \\
\hline & & Mean & SD & Mean & SD & Mean & SD & Mean & SD \\
\hline \multirow[t]{3}{*}{$10-11$} & Females & 41.53 & 23.57 & 65.73 & 11.26 & 5.15 & 13.7 & 14.59 & 21.59 \\
\hline & Males & 41.75 & 19.72 & 68.7 & 11.65 & 6.71 & 15.01 & 22.79 & 24.77 \\
\hline & Total & 41.63 & 21.79 & 67.17 & 11.54 & 5.9 & 14.36 & 18.54 & 23.53 \\
\hline \multirow[t]{3}{*}{$12-13$} & Females & 39.61 & 21.54 & 68.66 & 11.27 & 8.9 & 16.29 & 18.59 & 23.25 \\
\hline & Males & 43.32 & 20.18 & 71.37 & 11 & 8.43 & 14.82 & 26.74 & 25.85 \\
\hline & Total & 41.53 & 20.92 & 70.06 & 11.2 & 8.65 & 15.54 & 22.79 & 24.94 \\
\hline \multirow[t]{3}{*}{$14-15$} & Females & 43.37 & 24.08 & 70 & 11.05 & 16.79 & 21.98 & 19.17 & 22.94 \\
\hline & Males & 45.41 & 21.37 & 71.93 & 10.96 & 15.65 & 21.34 & 29.67 & 27.77 \\
\hline & Total & 44.37 & 22.79 & 70.96 & 11.04 & 16.23 & 21.66 & 24.35 & 25.95 \\
\hline \multirow[t]{3}{*}{$16-17$} & Females & 41.11 & 22.59 & 71.58 & 11.27 & 24.92 & 25.2 & 18.99 & 21.32 \\
\hline & Males & 43.32 & 20.27 & 74.2 & 9.94 & 30.47 & 27.02 & 32.35 & 26.32 \\
\hline & Total & 42.2 & 21.49 & 72.88 & 10.7 & 27.65 & 26.24 & 25.55 & 24.81 \\
\hline \multirow[t]{3}{*}{$18-19$} & Females & 45.14 & 23.48 & 70.68 & 11.31 & 30.60 & 26.63 & 21.38 & 21.99 \\
\hline & Males & 44.32 & 20.51 & 75.70 & 11.05 & 41.58 & 28.81 & 30.22 & 26.28 \\
\hline & Total & 44.73 & 22.01 & 73.25 & 11.44 & 36.24 & 28.27 & 25.92 & 24.66 \\
\hline \multirow[t]{3}{*}{$20-21$} & Females & 38.90 & 22.97 & 72.36 & 11.18 & 34.02 & 28.36 & 15.30 & 18.09 \\
\hline & Males & 44.98 & 22.81 & 75.52 & 10.53 & 47.84 & 28.56 & 29.77 & 25.78 \\
\hline & Total & 41.83 & 23.06 & 73.89 & 10.97 & 40.76 & 29.25 & 22.35 & 23.30 \\
\hline \multirow[t]{3}{*}{$22-23$} & Females & 41.58 & 23.33 & 70.89 & 10.63 & 34.14 & 27.08 & 15.91 & 19.30 \\
\hline & Males & 46.49 & 21.67 & 75.85 & 10.90 & 49.55 & 28.63 & 26.07 & 24.25 \\
\hline & Total & 43.92 & 22.66 & 73.27 & 11.03 & 41.45 & 28.84 & 20.73 & 22.34 \\
\hline \multirow[t]{3}{*}{$24-25$} & Females & 38.40 & 23.21 & 71.34 & 12.41 & 38.13 & 29.01 & 14.81 & 17.22 \\
\hline & Males & 42.90 & 22.49 & 74.27 & 11.46 & 52.06 & 27.50 & 25.82 & 23.27 \\
\hline & Total & 40.68 & 22.92 & 72.82 & 12.01 & 45.09 & 29.07 & 20.32 & 21.17 \\
\hline \multirow[t]{3}{*}{$26-27$} & Females & 39.77 & 21.39 & 72.18 & 10.52 & 41.49 & 29.25 & 15.46 & 21.43 \\
\hline & Males & 44.92 & 24.09 & 75.53 & 10.88 & 52.32 & 27.63 & 21.29 & 22.66 \\
\hline & Total & 42.38 & 22.90 & 73.86 & 10.81 & 46.89 & 28.92 & 18.36 & 22.20 \\
\hline \multirow[t]{3}{*}{$28-30$} & Females & 33.69 & 22.81 & 71.68 & 10.76 & 34.97 & 28.69 & 10.71 & 17.29 \\
\hline & Males & 38.92 & 23.14 & 75.41 & 9.82 & 51.67 & 28.90 & 22.56 & 23.83 \\
\hline & Total & 36.26 & 23.09 & 73.46 & 10.48 & 42.92 & 29.94 & 16.35 & 21.47 \\
\hline
\end{tabular}

Age is continuous in the analyses but grouped here to save space. Stoplight and BART are behavioral measures of risk taking propensity, health (drinking alcohol, getting in the car with a drunk driver, smoking cigarettes, and having unprotected sex) and antisocial (vandalizing, stealing, fighting, walking through a dangerous neighborhood, and threatening someone) risks are self-report scales. Possible range of values for all risk items is $0-100$

\section{Full Sample Analyses}

Both the linear and quadratic terms for age (age and age ${ }^{2}$, respectively) were significant for both measures of risktaking propensity (Stoplight and BART) and both measures of self-reported risk taking (health and antisocial risk scales) (see Table 4 for regression coefficients). Risk behavior on the Stoplight, BART, and self-reported antisocial risk taking generally demonstrated an inverted-U shape pattern across age groups, with adolescents evincing the highest risk scores. In contrast, self-reported health risk behaviors increased steeply across age groups before plateauing in the mid-20s. Figure 1 illustrates the age trends in risk taking for each of the four measures.

Risk taking was significantly greater among males than females for all tasks (see Table 4). Gender only moderated the linear effect of age for health risk taking $(\mathrm{B}=1.275$, SE $(B)=.363, \beta=.246, p<.001)$, such that increases in risk taking across age groups were steeper among males $(\mathrm{B}=3.138, \mathrm{SE}(\mathrm{B})=.083, \beta=.621, p<.001)$ than females $(\mathrm{B}=2.122, \mathrm{SE}(\mathrm{B})=.078, \beta=.479, p<.001)$. 
Table 4 Regression results for risk taking propensity and selfreported risk taking in the full sample

\begin{tabular}{|c|c|c|c|c|c|c|c|}
\hline \multirow[b]{2}{*}{ Risk Measure } & \multirow[b]{2}{*}{ Variable } & \multicolumn{3}{|c|}{ Linear model } & \multicolumn{3}{|c|}{ Quadratic model } \\
\hline & & $\mathrm{B}$ & SE (B) & Std. $\beta$ & $\mathrm{B}$ & SE (B) & Std. $\beta$ \\
\hline \multirow[t]{3}{*}{ Stoplight } & Gender & 2.51 & 0.581 & 0.057 & 2.504 & 0.581 & 0.056 \\
\hline & Age & $-\mathbf{0 . 1 6 3}$ & 0.054 & -0.043 & 0.69 & 0.186 & 0.184 \\
\hline & $\mathrm{Age}^{2}$ & & & & -0.048 & 0.01 & -0.238 \\
\hline \multirow[t]{3}{*}{ BART } & Gender & 3.091 & 0.308 & 0.135 & 3.068 & 0.305 & 0.134 \\
\hline & Age & 0.328 & 0.026 & 0.170 & .987 & 0.089 & 0.511 \\
\hline & $\mathrm{Age}^{2}$ & & & & $-\mathbf{0 . 0 3 7}$ & 0.005 & -0.357 \\
\hline \multirow[t]{3}{*}{ Health Risk } & Gender & 6.071 & 0.630 & 0.107 & 5.996 & 0.619 & 0.106 \\
\hline & Age & 2.607 & 0.059 & 0.546 & 5.009 & 0.192 & 1.049 \\
\hline & $\mathrm{Age}^{2}$ & & & & -0.136 & 0.011 & -0.526 \\
\hline \multirow[t]{3}{*}{ Antisocial Risk } & Gender & 10.386 & 0.645 & 0.216 & 10.339 & 0.643 & 0.215 \\
\hline & Age & -0.012 & 0.053 & -0.003 & 1.521 & 0.193 & 0.375 \\
\hline & $\mathrm{Age}^{2}$ & & & & -0.087 & 0.01 & -0.395 \\
\hline
\end{tabular}

Stoplight and BART are behavioral measures of risk taking propensity. Health and Antisocial Risk are selfreport scales. Values represent regression coefficients for the linear and quadratic models tested separately for each measure so that the linear term may be interpreted both independent of the quadratic term and as the instantaneous rate of change in the presence of a quadratic effect. Covariates (country, intellectual ability, and parental education) were omitted from the table to save space. Gender is dichotomous: $0=$ Female and 1 $=$ Male. Statistics for significant interactions among the age terms and gender are reported in Results. Unstandardized regression coefficients in bold are significant at $p<.05$

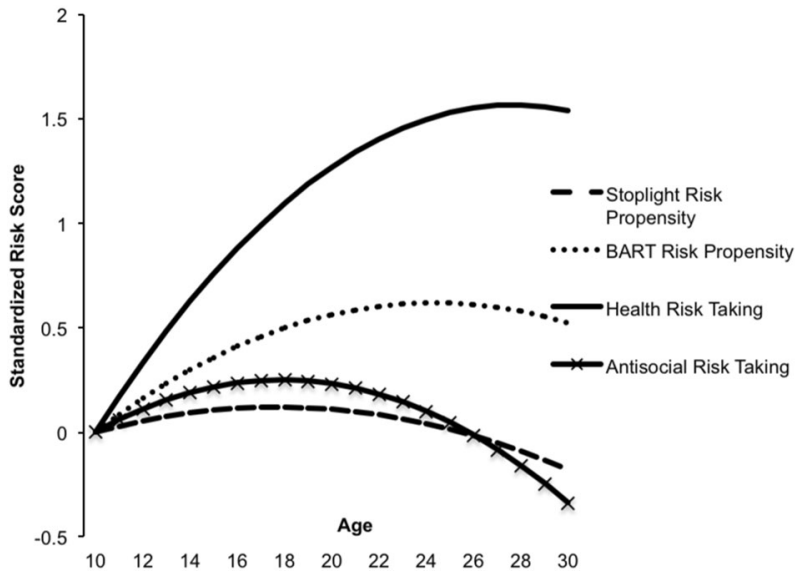

Fig. 1 Age differences in risk taking propensity and real-world risk taking. Note: Self-reported health risk taking $=$ drinking alcohol, getting in the car with a drunk driver, smoking cigarettes, and having unprotected sex; self-reported antisocial risk taking = vandalizing, stealing, fighting, walking through a dangerous neighborhood, and threatening someone. The model was estimated separately for each measure. Slopes represent Y-standardized estimated regression coefficients (centered at the slope for 10-year-olds) for age and age ${ }^{2}$ adjusted for country, gender, parental education, and intellectual ability. Slopes were standardized specifically for this figure so that measures could be interpreted on the same scale. The quadratic effect of age is significant for all measures

\section{Within-Country Analyses}

Chi-Square difference tests indicated that the free model was a better fit to the data than the fixed model (for
Stoplight, $\chi_{\text {fixed }}^{2}(70)=242.97, p<.001 ; \chi_{\text {free }}^{2}(20)=53.1$, $p<.001 ; \Delta \chi^{2}(\Delta d f=50)=189.87, p<.01$; for BART, $\chi_{\text {fixed }}^{2}(70)=229.68, p<.001 ; \chi_{\text {free }}^{2}(20)=77.99, p<.001$; $\Delta \chi^{2}(\Delta d f=50)=151.69, p<.01$; for self-reported health risk behavior, $\chi_{\text {fixed }}^{2}(70)=508.3, p<.001 ; \chi_{\text {free }}^{2}(20)=$ 28.77, $p=.092 ; \Delta \chi^{2}(\Delta d f=50)=479.53, p<.01$; and self-reported antisocial risk behavior, $\chi^{2}$ fixed $(70)=292.16$, $p<.001 ; \chi^{2}$ free $(20)=50.24, p<.001 ; \Delta \chi^{2}(\Delta d f=50)=$ $241.92, p<.01)$. Given our primary interest in risk behavior across age groups, we re-ran the multi-group analyses without the interactions between gender and age (or age ${ }^{2}$ ). In the free model, intellectual ability, parental education, and gender were held constant and age and $\mathrm{age}^{2}$ were free to vary across countries. In the fixed model, all parameters were constrained to be equal across countries. Results still indicated that the free model was a better fit to the data (for Stoplight, $\quad \chi_{\text {fixed }}^{2}(50)=209.93, \quad p<.001 ; \quad \chi_{\text {free }}^{2}(30)=$ $170.98, p<.001 ; \Delta \chi^{2}(\Delta d f=20)=39.95, p<.01$; for BART, $\chi_{\text {fixed }}^{2}(50)=198.73, p<.001 ; \chi_{\text {free }}^{2}(30)=114.95$, $p<.001 ; \Delta \chi^{2}(\Delta d f=20)=83.78, p<.01$; for self-reported health risk behavior, $\chi_{\text {fixed }}^{2}(50)=483.71, \quad p<.001$; $\chi_{\text {free }}^{2}(30)=70.17, p<.001 ; \Delta \chi^{2}(\Delta d f=20)=413.54, p$ $<.01$; and self-reported antisocial risk behavior, $\chi_{\text {fixed }}^{2}(50)$ $=258.63, p<.001 ; \chi^{2}$ free $(30)=152.72, p<.001 ; \Delta \chi^{2}$ $(\Delta d f=20)=105.91, p<.01)$. Based on these results, the full model (including the interactions with gender) was tested separately within each country for each measure of risk taking. 
Table 5 Regression results for within-country analyses of the stoplight task (risk propensity)

\begin{tabular}{|c|c|c|c|c|c|c|c|}
\hline \multirow[b]{2}{*}{ Country } & \multirow[b]{2}{*}{ Variable } & \multicolumn{3}{|c|}{ Linear model } & \multicolumn{3}{|c|}{ Quadratic model } \\
\hline & & B & SE (B) & Std. $\beta$ & B & SE (B) & Std. $\beta$ \\
\hline \multirow[t]{3}{*}{ China } & Gender & -0.903 & 1.996 & -0.021 & -1.041 & 1.998 & -0.024 \\
\hline & Age & -0.195 & 0.196 & -0.053 & 1.31 & 0.561 & 0.359 \\
\hline & $\mathrm{Age}^{2}$ & & & & -0.083 & 0.03 & -0.423 \\
\hline \multirow[t]{3}{*}{ Colombia } & Gender & 5.527 & 1.749 & 0.148 & 5.525 & 1.755 & 0.148 \\
\hline & Age & -0.36 & 0.144 & -0.115 & -0.535 & 0.511 & -0.17 \\
\hline & $\mathrm{Age}^{2}$ & & & & 0.01 & 0.029 & 0.059 \\
\hline \multirow[t]{3}{*}{ Cyprus } & Gender & 3.682 & 2.609 & 0.083 & 3.842 & 2.623 & 0.087 \\
\hline & Age & -0.237 & 0.244 & -0.063 & 0.666 & 0.86 & 0.178 \\
\hline & $\mathrm{Age}^{2}$ & & & & -0.046 & 0.045 & -0.249 \\
\hline \multirow[t]{3}{*}{ India } & Gender & 3.77 & 2.661 & 0.069 & 3.58 & 2.652 & 0.066 \\
\hline & Age & -0.691 & 0.223 & -0.147 & 0.985 & 0.84 & 0.21 \\
\hline & $\mathrm{Age}^{2}$ & & & & -0.09 & 0.043 & -0.373 \\
\hline \multirow[t]{3}{*}{ Italy } & Gender & -0.71 & 1.501 & -0.02 & -0.627 & 1.494 & -0.018 \\
\hline & Age & -0.114 & 0.144 & -0.039 & 1.335 & 0.436 & 0.455 \\
\hline & $\mathrm{Age}^{2}$ & & & & -0.083 & 0.026 & -0.515 \\
\hline \multirow[t]{3}{*}{ Jordan } & Gender & 21.862 & 1.927 & 0.524 & 21.651 & 1.932 & 0.519 \\
\hline & Age & 0.318 & 0.168 & 0.091 & 1.005 & 0.574 & 0.287 \\
\hline & $\mathrm{Age}^{2}$ & & & & -0.038 & 0.03 & -0.207 \\
\hline \multirow[t]{3}{*}{ Kenya } & Gender & -7.539 & 2.397 & -0.146 & -7.577 & 2.392 & -0.147 \\
\hline & Age & -0.077 & 0.211 & -0.018 & 1.108 & 0.738 & 0.253 \\
\hline & $\mathrm{Age}^{2}$ & & & & -0.065 & 0.039 & -0.28 \\
\hline \multirow[t]{3}{*}{ Philippines } & Gender & 2.451 & 1.883 & 0.057 & 2.369 & 1.879 & 0.056 \\
\hline & Age & $-\mathbf{0 . 5 3 7}$ & 0.167 & -0.149 & 0.591 & 0.556 & 0.164 \\
\hline & $\mathrm{Age}^{2}$ & & & & -0.065 & 0.03 & -0.325 \\
\hline \multirow[t]{3}{*}{ Sweden } & Gender & 1.287 & 1.857 & 0.034 & 1.277 & 1.861 & 0.034 \\
\hline & Age & 0.151 & 0.177 & 0.046 & -0.068 & 0.582 & -0.021 \\
\hline & $\mathrm{Age}^{2}$ & & & & 0.012 & 0.034 & 0.07 \\
\hline \multirow[t]{3}{*}{ Thailand } & Gender & 1.953 & 1.901 & 0.047 & 1.959 & 1.899 & 0.047 \\
\hline & Age & 0.174 & 0.184 & 0.047 & 0.254 & 0.61 & 0.069 \\
\hline & $\mathrm{Age}^{2}$ & & & & -0.005 & 0.036 & -0.023 \\
\hline \multirow[t]{3}{*}{ US } & Gender & 1.04 & 1.652 & 0.027 & 1.485 & 1.627 & 0.039 \\
\hline & Age & -0.203 & 0.135 & -0.064 & 1.292 & 0.53 & 0.406 \\
\hline & $\mathrm{Age}^{2}$ & & & & -0.086 & 0.03 & -0.487 \\
\hline
\end{tabular}

Values represent regression coefficients for the linear and quadratic models tested separately for each country so that the linear term may be interpreted both independent of the quadratic term and as the instantaneous rate of change in the presence of a quadratic effect. Covariates (parental education and intellectual ability) were omitted from to save space. Gender is dichotomous: $0=$ Female and $1=$ Male. Statistics for significant interactions among the age terms and gender are reported in Results. Unstandardized regression coefficients in bold are significant at $p<.05$

\section{Stoplight}

Risk taking on the Stoplight followed a negative linear (declining) trajectory across age groups in Colombia, a quadratic (inverted-U shape) pattern across age groups in India, Jordan (males only; see below) and the Philippines, and both a linear and quadratic pattern across age groups in China, Italy, and the US. Risk taking was not associated with age in Cyprus, Kenya, Sweden, and Thailand (see
Table 5 for regression coefficients). The slopes across age groups and country (see Fig. 2) indicated that in most countries, risk taking on the Stoplight increased modestly across the early adolescent groups before declining among late adolescents and young adults.

There was a main effect of gender in Colombia, Jordan, and Kenya. In Colombia and Jordan, risk taking was higher among males than females, but in Kenya, risk taking was higher among females (see Table 5). In all other countries, 


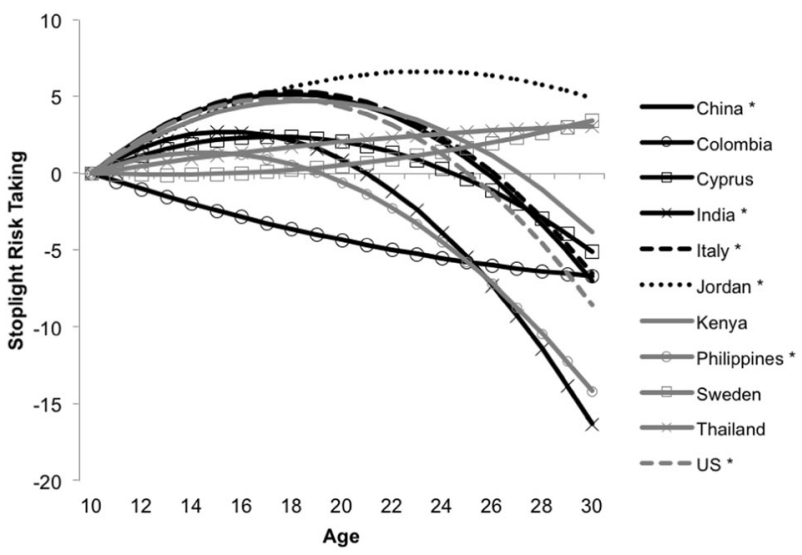

Fig. 2 Age differences in risk taking propensity on the Stoplight across countries. Slopes represent estimated regression coefficients (centered at the slope for 10-year-olds) for age and age ${ }^{2}$ adjusted for gender, parental education, and intellectual ability. The model was estimated separately for each country. * Countries for which there was a significant quadratic effect of age

mean levels of risk taking did not differ between males and females. In Jordan only, gender moderated the quadratic effect of age $(\mathrm{B}=-.155 ; \mathrm{SE}(\mathrm{B})=.058 ; \beta=-.657, p$ $<.05)$. Follow-up analyses indicated a quadratic effect of age among Jordanian males $(\mathrm{B}=-.109 ; \mathrm{SE}(\mathrm{B})=.044 ; \beta$ $=-.605, p<.05)$, but not females.

\section{Bart}

Risk taking on the BART task followed a positive linear (increasing) pattern across age groups in Kenya and Thailand, and both a linear and quadratic pattern across age groups in China, Colombia, Cyprus, India, Jordan, the Philippines, Sweden, and the US. Risk taking was not associated with age in Italy (see Table 6 for regression coefficients). In most countries, risk taking increased across the adolescent age groups and peaked among early adults before declining (see Fig. 3).

Results indicated a main effect of gender in all countries except Kenya and India, where mean levels of risk taking did not differ between males and females. In the countries for which there was a main effect of gender, risk taking was always greater among males than females (see Table 6). In Jordan only, gender moderated the quadratic effect of age $(\mathrm{B}=.125 ; \mathrm{SE}(\mathrm{B})=.038 ; \beta=.8, p<.05)$. Follow-up analyses indicated no effect of age among Jordanian males, but a quadratic effect of age among females $(\mathrm{B}=-.126$; SE $(\mathrm{B})=.031 ; \beta=-.947, p<.05)$.

\section{Self-reported health risk taking}

Self-reported health risk taking followed a positive linear (increasing) pattern across age groups in China, India,
Jordan, and Kenya, and both a linear and quadratic pattern in Colombia, Cyprus, Italy, the Philippines, Sweden, Thailand, and the US (see Table 7). The slopes (see Fig. 4) revealed that in essentially all countries, health risk behaviors continued increasing into the early adult age groups.

Results indicated a main effect of gender in China, Cyprus, India, Italy, Jordan, the Philippines, and Thailand, such that risk taking was greater among males than females. In Colombia, Kenya, Sweden, and the US, mean levels of health risk taking did not differ between males and females (see Table 7). Gender moderated the linear effect of age in China $(\mathrm{B}=2.928 ; \mathrm{SE}(\mathrm{B})=.966 ; \beta=.655, p<.05)$ and Kenya $(\mathrm{B}=2.742 ; \mathrm{SE}(\mathrm{B})=1.312 ; \beta=.665, p<.05)$. In China, age-related increases in risk taking were steeper among males than females. In Kenya, the linear effect of age was significant only among males $(\mathrm{B}=1.491$, SE (B) $=.271, \beta=.381, p<.05$ ), but not females.

\section{Self-reported antisocial risk taking}

Self-reported antisocial risk taking followed a negative linear (decreasing) pattern across age groups in China and Kenya, a quadratic (inverted-U shape) pattern in Thailand (females only; see below) and the Philippines, and both a linear and quadratic pattern in Colombia, India, Italy, Jordan, the Philippines, Sweden, and the US, and (see Table 8 for regression coefficients). Age was not associated with antisocial risk taking in Cyprus. The slopes (see Fig. 5) revealed that in most countries, antisocial risk taking increased into the late adolescent age groups and began declining among the early adults.

All countries evinced a main effect of gender, with greater risk taking among males than females (see Table 8). In Kenya, gender moderated the linear $(\mathrm{B}=5.194, \mathrm{SE}(\mathrm{B})$ $=1.55, \beta=1.033, p<.05)$ and quadratic $(\mathrm{B}=-.221, \mathrm{SE}$ $(\mathrm{B})=.078, \beta=-.718, p<.05)$ effects of age in that risk taking was associated with age for males $\left(\mathrm{B}_{\text {linear }}=3.483 ; \mathrm{SE}\right.$ $\left(\mathrm{B}_{\text {linear }}\right)=1.091 ; \beta_{\text {linear }}=.761, p_{\text {linear }}<.05 ; \mathrm{B}_{\text {quad }}=-.178$; $\left.\mathrm{SE} \quad\left(\mathrm{B}_{\text {quad }}\right)=.056 ; \beta_{\text {quad }}=-.745, p_{\text {quad }}<.05\right)$ but not females. In Thailand, gender moderated the quadratic effect of age $(\mathrm{B}=.135, \mathrm{SE}(\mathrm{B})=.067, \beta=.462, p<.05)$ such that the quadratic effect was significant for females $(\mathrm{B}=-.118$, $\mathrm{SE}(\mathrm{B})=.047, \beta=-.540, p<.05)$, but not males.

\section{Cross-national similarity in risk-taking propensity and self-reported risk-taking}

Chi-Square difference tests indicated that both constrained models for risk-taking propensity and self-reported risk taking yielded significantly worse model fit compared to the free model (which was just-identified) (for the propensity measures, $\Delta \chi^{2}(\Delta d f=100)=393.5, p<.01$; for the selfreport measures, $\left.\left.\Delta \chi^{2}(\Delta d f=100)=760.93\right), p<.01\right)$. 
Table 6 Regression results for within-country analyses of the bart task (risk propensity)

\begin{tabular}{|c|c|c|c|c|c|c|c|}
\hline \multirow[b]{2}{*}{ Country } & \multirow[b]{2}{*}{ Variable } & \multicolumn{3}{|c|}{ linear model } & \multicolumn{3}{|c|}{ Quadratic model } \\
\hline & & $\mathrm{B}$ & SE (B) & Std. $\beta$ & B & SE (B) & Std. $\beta$ \\
\hline \multirow[t]{3}{*}{ China } & Gender & 3.528 & 0.814 & 0.178 & 3.427 & 0.793 & 0.172 \\
\hline & Age & 0.407 & 0.079 & 0.242 & 1.54 & 0.26 & 0.914 \\
\hline & $\mathrm{Age}^{2}$ & & & & -0.063 & 0.013 & -0.689 \\
\hline \multirow[t]{3}{*}{ Colombia } & Gender & 2.763 & 0.867 & 0.14 & 2.728 & 0.862 & 0.139 \\
\hline & Age & 0.141 & 0.07 & 0.086 & 0.75 & 0.242 & 0.456 \\
\hline & $\mathrm{Age}^{2}$ & & & & $-\mathbf{0 . 0 3 6}$ & 0.013 & -0.39 \\
\hline \multirow[t]{3}{*}{ Cyprus } & Gender & 4.735 & 1.138 & 0.234 & 4.914 & 1.131 & 0.243 \\
\hline & Age & 0.03 & 0.1 & 0.018 & 0.977 & 0.37 & 0.572 \\
\hline & $\mathrm{Age}^{2}$ & & & & -0.049 & 0.018 & -0.575 \\
\hline \multirow[t]{3}{*}{ India } & Gender & 1.84 & 1.098 & 0.077 & 1.691 & 1.09 & 0.071 \\
\hline & Age & 0.31 & 0.101 & 0.15 & 0.998 & 0.33 & 0.483 \\
\hline & $\mathrm{Age}^{2}$ & & & & $-\mathbf{0 . 0 3 7}$ & 0.018 & -0.349 \\
\hline \multirow[t]{3}{*}{ Italy } & Gender & 2.969 & 0.889 & 0.143 & 2.963 & 0.889 & 0.143 \\
\hline & Age & 0.151 & 0.084 & 0.086 & 0.036 & 0.274 & 0.021 \\
\hline & $\mathrm{Age}^{2}$ & & & & 0.007 & 0.015 & 0.068 \\
\hline \multirow[t]{3}{*}{ Jordan } & Gender & 10.216 & 1.284 & 0.369 & 9.935 & 1.261 & 0.358 \\
\hline & Age & 0.58 & 0.123 & 0.248 & 1.787 & 0.357 & 0.764 \\
\hline & $\mathrm{Age}^{2}$ & & & & -0.067 & 0.019 & -0.546 \\
\hline \multirow[t]{3}{*}{ Kenya } & Gender & 0.157 & 1.157 & 0.006 & 0.12 & 1.166 & 0.005 \\
\hline & Age & 0.452 & 0.095 & 0.208 & 0.694 & 0.359 & 0.318 \\
\hline & Age2 & & & & -0.013 & 0.018 & -0.115 \\
\hline \multirow[t]{3}{*}{ Philippines } & Gender & 2.666 & 0.841 & 0.132 & 2.601 & 0.836 & 0.129 \\
\hline & Age & 0.372 & 0.071 & 0.217 & 1.21 & 0.24 & 0.708 \\
\hline & $\mathrm{Age}^{2}$ & & & & -0.048 & 0.013 & -0.511 \\
\hline \multirow[t]{3}{*}{ Sweden } & Gender & 2.675 & 0.9 & 0.151 & 2.725 & 0.897 & 0.154 \\
\hline & Age & -0.023 & 0.077 & -0.015 & 0.621 & 0.257 & 0.4 \\
\hline & $\mathrm{Age}^{2}$ & & & & -0.036 & 0.013 & -0.436 \\
\hline \multirow[t]{3}{*}{ Thailand } & Gender & 2.753 & 0.935 & 0.133 & 2.761 & 0.933 & 0.133 \\
\hline & Age & 0.21 & 0.088 & 0.115 & 0.575 & 0.3 & 0.316 \\
\hline & $\mathrm{Age}^{2}$ & & & & -0.022 & 0.017 & -0.211 \\
\hline \multirow[t]{3}{*}{ US } & Gender & 1.642 & 1.008 & 0.068 & 1.887 & 1.012 & 0.079 \\
\hline & Age & 0.583 & 0.091 & 0.288 & 1.607 & 0.308 & 0.794 \\
\hline & $\mathrm{Age}^{2}$ & & & & -0.059 & 0.017 & -0.525 \\
\hline
\end{tabular}

Values represent regression coefficients for the linear and quadratic models tested separately for each country so that the linear term may be interpreted both independent of the quadratic term and as the instantaneous rate of change in the presence of a quadratic effect. Covariates (parental education and intellectual ability) were omitted from the table to save space. Gender is dichotomous: $0=$ Female and $1=$ Male. Statistics for significant interactions among the age terms and gender are reported in Results. Unstandardized regression coefficients in bold are significant at $p<.05$
However, the $\chi^{2}$ model fit statistic was notably lower (suggesting better fit) for the propensity-constrained model than the self-report-constrained model. Further, comparison of other fit indices (Kline 2011) for the propensityconstrained and self-report-constrained models indicated notably worse model fit for the self-report-constrained model (for propensity measures, RMSEA $=.08(90 \% \mathrm{CI}$ : $.07-.09), \mathrm{CFI}=.93$; for self-report measures, RMSEA
$=.12(90 \%$ CI: $.11-.13), \mathrm{CFI}=.85)$. Ultimately, results pointed to greater cross-national similarity in the effect of age on risk taking for the risk propensity measures.

\section{Summary of findings}

Risk taking followed the hypothesized inverted-U pattern across age groups in 6 out of 11 countries on the Stoplight, 


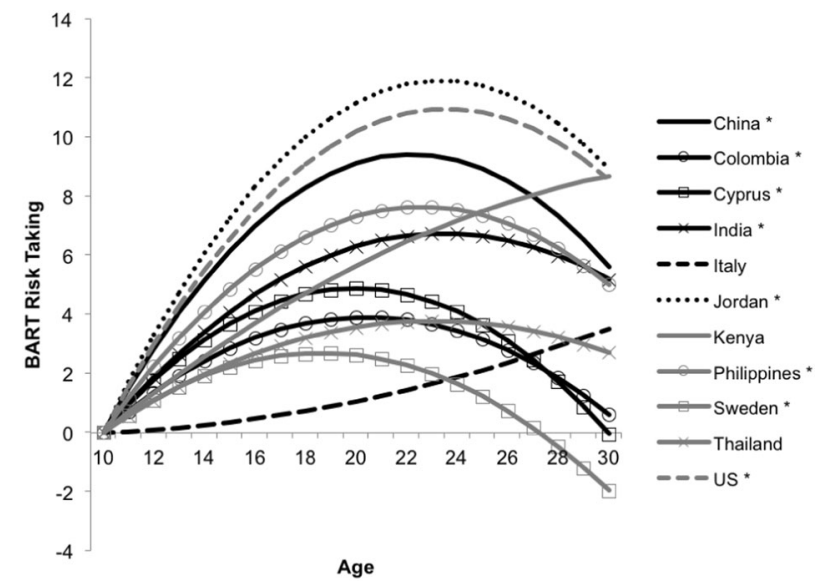

Fig. 3 Age differences in risk taking propensity on the BART across countries. Slopes represent estimated regression coefficients (centered at slope for 10-year-olds) for age and age ${ }^{2}$ adjusted for gender, parental education, and intellectual ability. The model was estimated separately for each country. * Countries for which there was a significant quadratic effect of age

8 out of 11 countries on the BART, 7 out of 11 countries on health risk behavior, and 9 out of the 11 countries on antisocial risk behavior. Although there were occasional exceptions (most consistently in Jordan and Kenya), the pattern of age differences did not differ between males and females, although at all ages, males almost always evinced greater risk taking than females. Furthermore, results indicated greater cross-national similarity in age patterns of risk taking propensity than age patterns of self-reported risk taking.

\section{Discussion}

Epidemiological studies of adolescent risk taking indicate that risky behavior (e.g., reckless driving) is among the leading causes of youth morbidity and mortality worldwide (WHO 2014). One explanation for this international public health problem is that adolescents demonstrate a heightened propensity, or inherent inclination, to take risks (Steinberg 2008). Findings from cross-sectional studies of risk behavior conducted in various parts of the world suggest that adolescents do generally take more risks than adults (Eisner 2002; Donato et al. 1995; Lotrean et al. 2010; Takakura et al. 2001). This seems to be particularly true with respect to antisocial risk behaviors, such as theft (Eisner 2002), whereas young adults demonstrate the highest levels of risk taking with respect to various health risks, such as binge drinking (Willoughby et al. 2013).

Important considerations in cross-cultural examinations of risk behavior are cultural differences in how risk is defined, in norms concerning adolescent behavior, and in opportunities to engage in various types of risky behavior.
One way to address this issue is to examine age patterns in risk-taking propensity using behavioral risk paradigms that minimize contextual confounds such as cultural norms and opportunities to engage in various types of risk taking. Comparing age patterns in risk-taking propensity across countries using behavioral paradigms helps clarify whether heightened risk taking in adolescence is a phenomenon that is observed across cultures, or whether this phenomenon is culturally variable.

The present study examined age patterns in risk taking across eleven countries (China, Colombia, Cyprus, India, Italy, Jordan, Kenya, the Philippines, Sweden, Thailand, and the US) using two behavioral tasks of risk-taking propensity and two self-report measures of real-world risk taking. Consistent with our first hypothesis, risk taking is generally highest in late adolescence. This inverted-U pattern of risk taking across age is evinced broadly across countries, despite variation in their cultural and economic contexts. In support of our second hypothesis, we find greater cross-national similarity in age patterns of risktaking propensity than in patterns of self-reported risk taking, supporting the notion that age differences in real-world risk taking are influenced by environmental forces that affect the expression of age differences in the propensity to behave recklessly. Nevertheless, it is clear that around the world, individuals' inclination to take risks, as well as their actual risky behavior, is higher in late adolescence and young adulthood than before or after.

Cross-sectional studies of age patterns in risk taking have yielded discordant findings with respect to whether risk taking peaks in adolescence or early adulthood (see Shulman et al. 2016 and Willoughby et al. 2013). Neuroscientifically-informed theories of adolescent risk taking, such as dual systems or maturational imbalance models (Casey et al. 2011; Steinberg 2008), predict that risk taking is highest in mid-adolescence to late adolescence, when heightened reward sensitivity has the potential to draw adolescents towards risky behaviors and overwhelm still-maturing self-regulatory abilities. However, because opportunities to engage in many health-risk behaviors, such as getting into a car with a drunk driver, increase with age, it is likely that adults demonstrate greater health-risk taking compared to adolescents. Consistent with this, real-world health risk taking reaches its apex at a later age (around early adulthood) than either risk-taking propensity (on the Stoplight and BART) or real-world antisocial risk taking. That some countries evince a peak in health-risk taking in early adulthood whereas others demonstrate a steady increase between adolescence and adulthood suggests differences in cultural norms that likely influence engagement in various risks. Binge drinking, for example, reaches its apex in early adulthood in the US (Willoughby et al. 2013), when most individuals are attending college, an 
Table 7 Regression results for within-country analyses of selfreported health risk behaviors

\begin{tabular}{|c|c|c|c|c|c|c|c|}
\hline \multirow[b]{2}{*}{ Country } & \multirow[b]{2}{*}{ Variable } & \multicolumn{3}{|c|}{ Linear model } & \multicolumn{3}{|c|}{ Quadratic model } \\
\hline & & $\mathrm{B}$ & SE (B) & Std. $\beta$ & B & $\mathrm{SE}(\mathrm{B})$ & Std. $\beta$ \\
\hline \multirow[t]{3}{*}{ China } & Gender & 7.598 & 1.792 & 0.154 & 7.563 & 1.789 & 0.154 \\
\hline & Age & 2.47 & 0.186 & 0.591 & 2.873 & 0.554 & 0.688 \\
\hline & $\mathrm{Age}^{2}$ & & & & -0.022 & 0.033 & -0.099 \\
\hline \multirow[t]{3}{*}{ Colombia } & Gender & 3.742 & 2.152 & 0.065 & 3.51 & 2.087 & 0.061 \\
\hline & Age & 2.638 & 0.189 & 0.547 & 6.661 & 0.576 & 1.38 \\
\hline & $\mathrm{Age}^{2}$ & & & & -0.238 & 0.035 & -0.879 \\
\hline \multirow[t]{3}{*}{ Cyprus } & Gender & 9.805 & 2.951 & 0.155 & 10.18 & 2.87 & 0.161 \\
\hline & Age & 3.329 & 0.243 & 0.616 & 6.388 & 0.736 & 1.182 \\
\hline & $\mathrm{Age}^{2}$ & & & & -0.159 & 0.038 & -0.589 \\
\hline \multirow[t]{3}{*}{ India } & Gender & 7.558 & 2.072 & 0.153 & 7.436 & 2.069 & 0.15 \\
\hline & Age & 2.262 & 0.192 & 0.528 & 2.941 & 0.636 & 0.687 \\
\hline & $\mathrm{Age}^{2}$ & & & & -0.037 & 0.037 & -0.166 \\
\hline \multirow[t]{3}{*}{ Italy } & Gender & 4.712 & 1.931 & 0.072 & 4.928 & 1.779 & 0.076 \\
\hline & Age & 4.017 & 0.181 & 0.733 & 9.097 & 0.54 & 1.66 \\
\hline & $\mathrm{Age}^{2}$ & & & & -0.293 & 0.033 & -0.967 \\
\hline \multirow[t]{3}{*}{ Jordan } & Gender & 14.136 & 2.091 & 0.342 & 13.917 & 2.114 & 0.337 \\
\hline & Age & 1.133 & 0.156 & 0.326 & 2.026 & 0.502 & 0.583 \\
\hline & $\mathrm{Age}^{2}$ & & & & -0.05 & 0.029 & -0.271 \\
\hline \multirow[t]{3}{*}{ Kenya } & Gender & 2.594 & 1.992 & 0.057 & 2.663 & 1.989 & 0.059 \\
\hline & Age & 0.857 & 0.177 & 0.225 & 0.392 & 0.643 & 0.103 \\
\hline & Age2 & & & & 0.025 & 0.033 & 0.126 \\
\hline \multirow[t]{3}{*}{ Philippines } & Gender & 6.884 & 1.936 & 0.132 & 6.785 & 1.91 & 0.13 \\
\hline & Age & 2.411 & 0.191 & 0.544 & 3.695 & 0.574 & 0.834 \\
\hline & $\mathrm{Age}^{2}$ & & & & -0.074 & 0.036 & -0.302 \\
\hline \multirow[t]{3}{*}{ Sweden } & Gender & 2.134 & 2.5 & 0.035 & 2.518 & 2.358 & 0.041 \\
\hline & Age & 3.138 & 0.221 & 0.581 & 8.415 & 0.667 & 1.557 \\
\hline & $\mathrm{Age}^{2}$ & & & & -0.295 & 0.04 & -1.027 \\
\hline \multirow[t]{3}{*}{ Thailand } & Gender & 9.064 & 2.09 & 0.151 & 9.105 & 2.069 & 0.151 \\
\hline & Age & 3.359 & 0.189 & 0.636 & 5.496 & 0.713 & 1.04 \\
\hline & $\mathrm{Age}^{2}$ & & & & -0.126 & 0.042 & -0.424 \\
\hline \multirow[t]{3}{*}{ US } & Gender & 0.779 & 1.86 & 0.014 & 1.615 & 1.83 & 0.029 \\
\hline & Age & 3.107 & 0.178 & 0.659 & 6.277 & 0.616 & 1.331 \\
\hline & $\mathrm{Age}^{2}$ & & & & -0.181 & 0.036 & -0.697 \\
\hline
\end{tabular}

Values represent regression coefficients for the linear and quadratic models tested separately for each country so that the linear term may be interpreted both independent of the quadratic term and as the instantaneous rate of change in the presence of a quadratic effect. Covariates (parental education and intellectual ability) were omitted to save space. Gender is dichotomous: $0=$ Female and $1=$ Male. Statistics for significant interactions among the age terms and gender are reported in Results. Unstandardized regression coefficients in bold are significant at $p<.05$ environment in which binge drinking and partying are common and much more prevalent than after college. Such a culture may be less prevalent in countries where individuals begin drinking during adolescence, in the presence of adults. Adolescent drinking in China, for example, is often accepted and at times encouraged by parents because it is considered an integral part of social communication in Chinese culture (Xing et al. 2006), similar to some of the drinking norms in European countries such as Italy (Donato et al. 1995). Patterns of drinking behavior among adolescents from these countries are likely to differ from the drinking patterns of adolescents from countries in which drinking is prohibited until age 21 .

We suspect that the observed age differences in antisocial risk taking demonstrate an inverted-U pattern more consistent with age patterns in risk-taking propensity than 


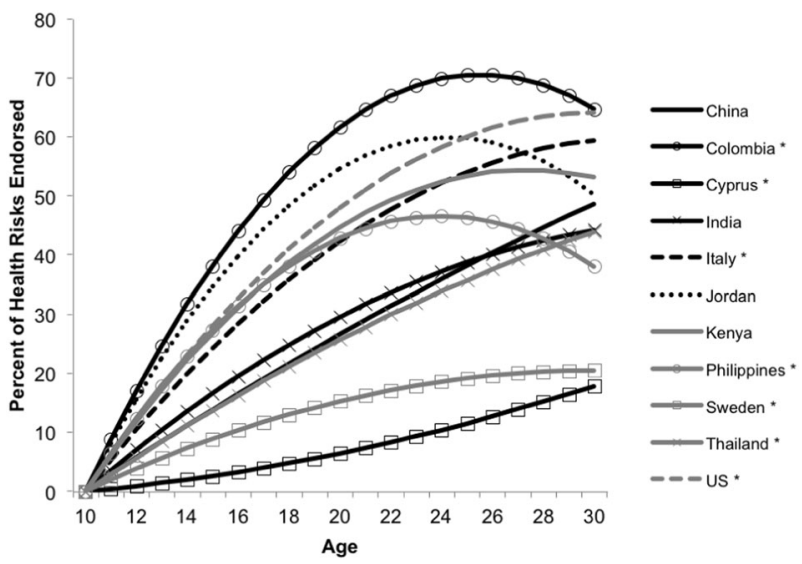

Fig. 4 Age patterns in self-reported health risk taking across countries. Values are percentage $(\%)$ of health risks (drinking alcohol, getting in the car with a drunk driver, smoking cigarettes, and having unprotected sex) endorsed. Slopes represent estimated regression coefficients (centered at the slope for 10-year-olds) for age and age ${ }^{2}$ adjusted for gender, parental education, and intellectual ability. The model was estimated separately for each country. * Countries for which there was a significant quadratic effect of age

health-risk taking because opportunities to engage in antisocial behavior are not as constrained as opportunities to take health risks. Further, the antisocial behaviors measured in our study are generally less serious acts (e.g., vandalism, shoplifting, fighting) that would not be observed exclusively among serious juvenile offenders. And indeed, within our sample, we see a wide range of endorsement in various antisocial risk behaviors (results not reported). Further, behaviors such as vandalizing a bathroom stall, stealing gum from a convenience store, or getting into a fight with peers are all actions that are less constrained than gaining access to alcohol or cigarettes. The age patterns in antisocial risk taking observed across countries are consistent with the "age-crime curve" that has been observed in many studies from various countries (cf., Eisner 2002). That age patterns in antisocial risk taking are more consistent with age patterns in risk-taking propensity rather than health-risk taking supports the general proposition that real world risk taking is a product of the interaction between inclination and opportunity.

Although we anticipated that age patterns in risk-taking propensity on the Stoplight and BART tasks would be comparable, we found some variability in age patterns in risk taking on these two tasks. We do not have an obvious explanation as to why this is the case. One possibility is that the underlying influences on BART performance comprise various factors that develop along different timetables. On the one hand, inflating the balloons nearly to the point of explosion may reflect sensation seeking, which should result in a peak in risk taking in mid- to late adolescence (Shulman et al. 2016). On the other hand, inflating the balloons to a greater extent may reflect greater self-control and a willingness to delay gratification (i.e., resisting the impulse to terminate inflation and cash out early for a smaller, but more immediate reward; Dahne et al. 2013), which should result in a later peak. In contrast, performance on the Stoplight task is driven mainly by sensation seeking and reward sensitivity, perhaps because the task is more affectively arousing compared to the BART (e.g., a driving simulation with loud music may be more exciting than simply watching a balloon inflate), and is unrelated to impulsivity (Steinberg et al. 2008). The lesson here is that although many different laboratory tasks are used to measure risk taking, in actuality, tasks that purport to assess the same construct may actually be tapping into different psychological traits related to risk taking.

This general point- that different measures of risk taking likely measure different phenomena-also applies to the distinction between laboratory measures, which in our view assess underlying inclinations, and self-reports of risky behavior in the real world, which reflect a combination of these underlying inclinations and the context in which these inclinations play out. Age was associated with health risk taking in all 11 countries included in this study, peaking during the mid or late 20 s. This pattern is largely consistent with previous developmental and cross-national studies of real-world risk taking that have found health-risk behaviors to increase into, and sometimes beyond, the 20s (e.g., Anderson Johnson et al. 2006; Eisner 2002; Plant et al. 2009; Willoughby et al. 2013). We view our finding of a relatively late peak in health-risk taking as clarifying, rather than contradicting, the notion that adolescence is a risker time than either childhood or adulthood. Inclinations to behave recklessly are strongest in adolescence, but opportunities to realize these inclinations are more plentiful in adulthood.

Consistent with previous work (Jessor et al. 2003; Lejuez et al. 2003; Plant et al. 2009; Takakura et al. 2001; WHO 2014), our analyses of gender differences indicate, as one might expect, that males generally engage in more risk taking than females. This finding was most consistently observed on measures of real-world risk taking as opposed to measures of risk-taking propensity. Previous studies using risky driving paradigms (e.g., Gardner and Steinberg 2005) and the BART task (e.g., Lejuez et al. 2003) have also failed to find gender differences. These findings strongly suggest that gender differences in real-world risk taking are more likely due to differences in the opportunities that males and females have to engage in these behaviors rather than gender differences in brain or psychosocial development. Consistent with this, there was little variation across countries in age patterns of risk taking between males and females. Although males and females differed in their mean levels of real-world risk taking, the fact that this 
Table 8 Regression results for within-country analyses of selfreported antisocial risk behaviors

\begin{tabular}{|c|c|c|c|c|c|c|c|}
\hline \multirow[b]{2}{*}{ Country } & \multirow[b]{2}{*}{ Variable } & \multicolumn{3}{|c|}{ Linear model } & \multicolumn{3}{|c|}{ Quadratic model } \\
\hline & & B & SE (B) & Std. $\beta$ & B & SE (B) & Std. $\beta$ \\
\hline \multirow[t]{3}{*}{ China } & Gender & 5.258 & 1.428 & 0.167 & 5.224 & 1.428 & 0.165 \\
\hline & Age & $-\mathbf{0 . 3 5 7}$ & 0.123 & -0.133 & 0.112 & 0.417 & 0.042 \\
\hline & $\mathrm{Age}^{2}$ & & & & -0.026 & 0.021 & -0.179 \\
\hline \multirow[t]{3}{*}{ Colombia } & Gender & 8.156 & 2.077 & 0.174 & 8.025 & 2.054 & 0.171 \\
\hline & Age & 0.315 & 0.166 & 0.08 & 2.626 & 0.543 & 0.67 \\
\hline & $\mathrm{Age}^{2}$ & & & & -0.137 & 0.031 & -0.621 \\
\hline \multirow[t]{3}{*}{ Cyprus } & Gender & 14.445 & 2.705 & 0.304 & 14.401 & 2.718 & 0.304 \\
\hline & Age & -0.32 & 0.239 & -0.079 & -0.673 & 0.757 & -0.166 \\
\hline & $\mathrm{Age}^{2}$ & & & & 0.018 & 0.039 & 0.091 \\
\hline \multirow[t]{3}{*}{ India } & Gender & 13.664 & 2.221 & 0.279 & 13.268 & 2.214 & 0.271 \\
\hline & Age & 0.434 & 0.183 & 0.102 & 2.638 & 0.615 & 0.623 \\
\hline & $\mathrm{Age}^{2}$ & & & & -0.119 & 0.034 & -0.544 \\
\hline \multirow[t]{3}{*}{ Italy } & Gender & 8.735 & 1.779 & 0.202 & 8.847 & 1.754 & 0.205 \\
\hline & Age & 0.452 & 0.155 & 0.124 & 2.804 & 0.489 & 0.769 \\
\hline & $\mathrm{Age}^{2}$ & & & & -0.136 & 0.026 & -0.673 \\
\hline \multirow[t]{3}{*}{ Jordan } & Gender & 30.814 & 2.793 & 0.519 & 30.553 & 2.799 & 0.515 \\
\hline & Age & 0.049 & 0.226 & 0.01 & 1.333 & 0.631 & 0.267 \\
\hline & $\mathrm{Age}^{2}$ & & & & -0.071 & 0.034 & -0.272 \\
\hline \multirow[t]{3}{*}{ Kenya } & Gender & 7.682 & 2.523 & 0.139 & 7.534 & 2.499 & 0.137 \\
\hline & Age & -0.432 & 0.198 & -0.093 & 0.597 & 0.787 & 0.129 \\
\hline & Age2 & & & & -0.056 & 0.039 & -0.23 \\
\hline \multirow[t]{3}{*}{ Philippines } & Gender & 9.4 & 2.023 & 0.191 & 9.282 & 2.017 & 0.189 \\
\hline & Age & -1.058 & 0.165 & -0.254 & 0.444 & 0.619 & 0.107 \\
\hline & $\mathrm{Age}^{2}$ & & & & -0.086 & 0.033 & -0.375 \\
\hline \multirow[t]{3}{*}{ Sweden } & Gender & 7.384 & 1.795 & 0.191 & 7.527 & 1.766 & 0.194 \\
\hline & Age & -0.092 & 0.147 & -0.027 & 2.021 & 0.478 & 0.595 \\
\hline & $\mathrm{Age}^{2}$ & & & & -0.118 & 0.024 & -0.654 \\
\hline \multirow[t]{3}{*}{ Thailand } & Gender & 4.549 & 2.103 & 0.1 & 4.568 & 2.098 & 0.1 \\
\hline & Age & -.318 & .165 & -.08 & 0.568 & 0.605 & 0.142 \\
\hline & $\mathrm{Age}^{2}$ & & & & -0.052 & 0.033 & -0.232 \\
\hline \multirow[t]{3}{*}{ US } & Gender & 7.977 & 1.778 & 0.181 & 8.462 & 1.77 & 0.192 \\
\hline & Age & 0.702 & 0.164 & 0.19 & 2.506 & 0.607 & 0.681 \\
\hline & $\mathrm{Age}^{2}$ & & & & -0.103 & 0.034 & -0.508 \\
\hline
\end{tabular}

Values represent regression coefficients for the linear and quadratic models tested separately for each country so that the linear term may be interpreted both independent of the quadratic term and as the instantaneous rate of change in the presence of a quadratic effect. Covariates (parental education and intellectual ability) were omitted from to save space. Gender is dichotomous: $0=$ Female and $1=$ Male. Statistics for significant interactions among the age terms and gender are reported in Results. Unstandardized regression coefficients in bold are significant at $p<.05$ behavior increases during adolescence and declines during the $20 \mathrm{~s}$ is true for both genders.

Perhaps the greatest strength of the present study is the large international sample of individuals ranging in age from 10 to 30 , which allowed us to examine age differences in risk taking throughout adolescence and into adulthood, as well as to examine the extent to which these age patterns are consistent across countries that vary in their social, political, cultural, and economic contexts. Furthermore, this study is one of the first to explore international variability in age patterns of both risk-taking propensity, using experimental tasks, and real-world risk taking, based on self-reports. Behavioral tasks assessing risk-taking propensity, which are commonly used in studies of risk behavior in Western samples (e.g., Kim-Spoon et al. 2016; Mitchell et al. 2008), have rarely been implemented in international samples. 


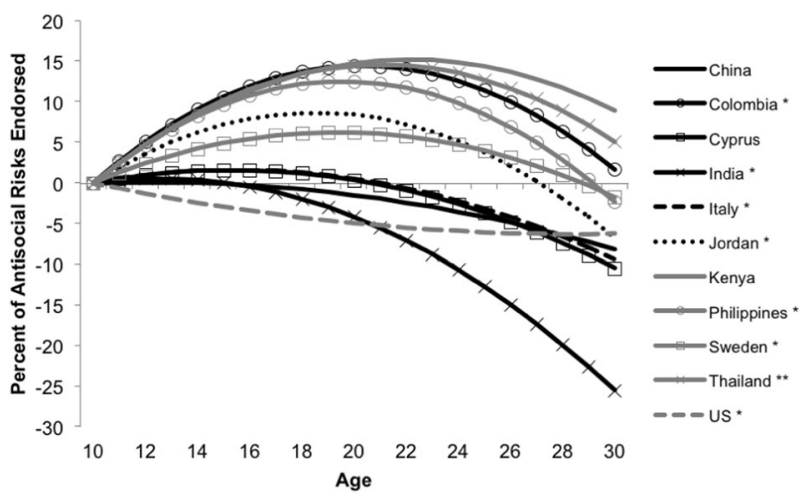

Fig. 5 Age patterns in self-reported antisocial risk taking across countries. Values are percentage (\%) of antisocial risks (vandalizing, stealing, fighting, walking through a dangerous neighborhood, and threatening someone) endorsed. Slopes represent estimated regression coefficients (centered at the slope 10-year-olds) for age and age $^{2}$ adjusted for gender, parental education, and intellectual ability. The model was estimated separately for each country. ${ }^{*}$ Countries for which there was a significant quadratic effect of age. ${ }^{* *}$ The quadratic effect is significant for females, but not males

Additionally, although epidemiological data on adolescent risk behavior exist (e.g., WHO 2014), our self-report scales offer a larger variety of risk behaviors, and our sample allows for age comparisons across a broader range of developmental periods. Including various risk measures afforded us the opportunity to explore the potential reasons for discrepancies in previous studies in the observed peak age of risk taking. The answer, it appears, is that different measures of risk taking yield slightly different age patterns.

Despite these strengths, this study was limited in a few ways. One limitation is that the risk propensity measures employed in this study did not include affective manipulations, which have been shown to influence adolescent risk behavior (cf., Shulman et al. 2016). Employing a task with an affective manipulation (such as real-time feedback or peer presence) might have yielded clearer differences in age patterns of risk taking between the behavioral tasks and the self-report measures, perhaps evincing an earlier peak on experimental tasks (e.g., Defoe et al. 2015; Shulman et al. 2016) — a strong possibility that warrants further study. Additionally, although we included two different measures of risk-taking propensity, it is clear, based on different age patterns evinced on the Stoplight and BART, that different tasks tap into different psychological phenomena, and that a more thorough examination of this issue will require more than just one pair of measures. Along similar lines, we acknowledge that the relatively greater cross-national similarity in age patterns of risk-taking propensity may be due to the fact that our propensity measures are derived from behavioral tasks, whereas our real-world measures are based on self-reports. Hence, we cannot be sure that the difference in cross-national homogeneity is substantive (i.e., propensity vs. actual risk taking), or methodological (i.e., behavioral vs. questionnaire). Finally, we note that our inferences about the peak age period in risk taking are tempered by the fact that they are based on means and estimated regression coefficients. We also acknowledge that our explanations for the observed differences in risk taking with respect to both age and culture are only speculative. It is critical for future work to explore various factors (e.g., economic wealth, public health practices, religion, and behavioral norms among adolescents) that are thought to influence risk taking within various countries. However, given the dearth of international research on age patterns in risk-taking propensity on lab tasks, and that epidemiological reports seldom use statistical tests to explore age patterns in risk behavior, we believe that our findings offer an important step forward in this field of research.

\section{Conclusion}

There are three key points to take from this study. First, and consistent with popular portrayals of adolescence, age patterns in risk taking are largely consistent across cultures, at least in the very general sense that risk taking rises in adolescence and subsequently declines in adulthood. This cross-national similarity is consistent with recent reports of cross-cultural comparability in age patterns of sensationseeking and self-regulation (Steinberg et al. 2017), two psychological phenomena thought to influence risky behavior (Duell et al. 2016). To the extent that adolescence is inherently a time when individuals are highly motivated to seek rewards but not entirely able to rein in these inclinations, some experimentation with risky activity may be unavoidable. Second, despite this broad conclusion about the vicissitudes of risk-taking propensity in adolescence and young adulthood, it is clear that the relation between age and risk taking is sensitive to the way risk taking is measured. Finally, and most important, the ways in which, and the extent to which, adolescents' propensities to engage in risky activity are manifested in real-world recklessness seems to vary across the cultural contexts in which these inclinations develop. Despite recent fascination with adolescent brain development in the scientific literature and popular press, it is essential to remind ourselves that age differences in behaviors that likely have strong roots in biology are ultimately shaped by the settings in which young people grow up.

Funding This research was supported by an award to Laurence Steinberg from the Klaus J. Jacobs Foundation and the Eunice Kennedy Shriver National Institute of Child Health and Human Development grant RO1-HD054805. 
Authors' Contributions ND conceived of the study, participated in its design, performed the statistical analyses, participated in interpretation of the data, and drafted the manuscript; LS conceived of the study, participated in its design and coordination, participated in interpretation of the data, and helped to draft the manuscript; GI participated in the study design and in interpretation of the data; JC gave final approval of the manuscript; $\mathrm{NC}$ was involved in the acquisition of the data and gave final approval of the manuscript; LD was involved in the acquisition of the data and gave final approval of the manuscript; KAD was involved in the acquisition of the data and gave final approval of the manuscript; KAF was involved in the acquisition of the data and gave final approval of the manuscript; JEL was involved in the acquisition of the data and gave final approval of the manuscript; PO was involved in the acquisition of the data and gave final approval of the manuscript; $\mathrm{CP}$ was involved in the acquisition of the data and gave final approval of the manuscript; ATS was involved in the acquisition of the data and gave final approval of the manuscript; ES was involved in the acquisition of the data and gave final approval of the manuscript; ST was involved in the acquisition of the data and gave final approval of the manuscript; LUT was involved in the acquisition of the data and gave final approval of the manuscript; LPA was involved in the acquisition of the data and gave final approval of the manuscript; SMA was involved in the acquisition of the data and gave final approval of the manuscript; HMST was involved in the acquisition of the data and gave final approval of the manuscript; LC was involved in the acquisition of the data and gave final approval of the manuscript; and DB was involved in the acquisition of the data and gave final approval of the manuscript. All authors read and approved the final manuscript.

\section{Compliance with Ethical Standards}

Conflicts of Interest The authors declare that they have no competing interests.

Ethical Approval All procedures performed in studies involving human participants were in accordance with the ethical standards of the institutional and/or national research committee and with the 1964 Helsinki declaration and its later amendments or comparable ethical standards.

Informed Consent Informed consent was obtained from all individual participants included in the study.

\section{References}

Benthin, A., Slovic, P. \& Severson, H. (1993). A Psychometric study of adolescent risk perception. Journal of Adolescence, 16(2), $153-168$

Anderson Johnson, C., Palmer, P. H., Chou, C. P., Pang, Z., Zhou, D., Dong, L., Xiang, H., Yang, P., \& Xu, H., et al. (2006). Tobacco use among youth and adults in Mainland China: The china seven cities study. Public Health, 120, 1156-1169. https://doi.org/10. 1016/j.puhe.2006.07.023.

Anteghini, M., Fonseca, H., Ireland, M., \& Blum, R. (2001). Health risk behaviors and associated risk protective factors among Brazilian adolescents in Santos, Brazil. Journal of Adolescent Health, 28, 295-302. https://doi.org/10.1016/S1054-139X(00)00197-X.

Casey, B. J., Jones, R., \& Somerville, L. (2011). Braking and accelerating of the adolescent brain. Journal of Research on Adolescence, 21, 21-33. https://doi.org/10.1111/j.1532-7795.2010.00712.x.
Choudhury, S. (2010). Culturing the adolescent brain: What can neuroscience learn from anthropology? Social Cognitive and Affective Neuroscience, 5, 159-167. https://doi.org/10.1093/scan/ nsp030.

Dahne, J., Richards, J. M., Ernst, M., Macpherson, L., \& Lejuez, C. W. (2013). Assessment of risk taking in addiction research. In J. MacKillop \& H. de Wit (Eds.), The Wiley-Blackwell Handbook of Addiction Psychopharmacology (pp. 209-231). Hoboken, NJ: Wiley-Blackwell. https://doi.org/10.1002/9781118384404.ch8

Defoe, I. N., Semon Dubas, J., Figner, B., \& van Aken, M. A. G. (2015). A meta-analysis on age differences in risky decision making: Adolescents versus children and adults. Psychological Bulletin, 141, 48-84. https://doi.org/10.1037/a0038088.

Donato, F., Monarca, S., Chiesa, R., Feretti, D., Modolo, M. A., \& Nardi, G. (1995). Patterns and covariates of alcohol drinking among high school students in 10 towns in Italy: A crosssectional study. Drug and Alcohol Dependence, 37, 59-69. https://doi.org/10.1016/0376-8716(94)01053-N.

Duell, N., Steinberg, L., Chein, J., Al-Hassan, S. M., Bacchini, D., Lei, C., Chaudhary, N., Di Giunta, L., \& Dodge, K. A., et al. (2016). Interaction of reward seeking and self-regulation in the prediction of risk taking: A cross-national test of the dual systems model. Developmental Psychology, 52, 1593-1605. https://doi.org/10. 1037/dev0000152.

Efron, B., \& Tibshirani, R. J. (1993). An Introduction to the Bootstrap. New York: Chapman \& Hall.

Eisner, M. (2002). Crime, problem drinking, and drug use: Patterns of problem behavior in cross-national perspective. The Annals of the American Academy of Political and Social Science, 580, 201-225.

Ellis, L., \& Walsh, A. (2003). Crime, delinquency, and intelligence: A review of the worldwide literature. In $\mathrm{H}$. Nyborg (Ed.), The Scientific Study of General Intelligence: Tribute to Arthur J. Jensen (pp. 343-365). Kidlington, Oxford: Elsevier Science Ltd. https://doi.org/10.1016/B978-008043793-4/50054-4.

Erkut, S. (2010). Developing Multiple Language Versions of Instruments for Intercultural Research. Child Development Perspectives 4(1), 19-24.

Fuller, E., Clifton, S., Field, N., Mercer. C. H., Prah, P., Macdowall, W., Mitchell, K., Sonnenberg, P., Johnson, A. M., \& Wellings, K. (2015). Natsal-3: Key findings from Scotland. http://natcen.ac.uk/ media/997277/NatSal-Scotland.pdf.

Gardner, M., \& Steinberg, L. (2005). Peer influence on risk taking, risk preference, and risky decision making in adolescence and adulthood: An experimental study. Developmental Psychology, 41, 625-635. https://doi.org/10.1037/0012-1649.41.4.625.

Hawley, P. H. (2011). The evolution of adolescence and the adolescence of evolution: The coming of age of humans and the theory about the forces that made them. Journal of Research on Adolescence, 21, 307-306. https://doi.org/10.1111/j.1532-7795.2010. 00732.x.

Hendriksen, E. S., Pettifor, A., Lee, S., Coates, T. J., \& Rees, H. V. (2007). Predictors of condom use among young adults in South Africa: The reproductive health and HIV research unit national youth survey. American Journal of Public Health, 97, 1-8. https://doi.org/10.2105/AJPH.2006.086009.

Hirschi, T., \& Gottfredson, M. R. (1983). Age and the explanation of crime. American Journal of Sociology, 89, 552-584.

Hindelang, M. J., Hirschi, T. \& Weis, J. G. (1981). Measuring delinquency. Beverly Hills, CA: Sage Publications.

Hofstede, G. (2011). Dimensionalizing cultures: The Hofstede model in context. Online Readings in Psychology \& Culture, 2, 1-26. https://doi.org/10.9707/2307-0919.1014.

Jessor, R., Turbin, M. S., Costa, F. M., Dong, Q., Zhang, H., \& Wang, C. (2003). Adolescent problem behavior in China and the United States: A cross-national study of psychosocial 
protective factors. Journal of Research on Adolescence, 13, 329-260.

Kim-Spoon, J., Kahn, R., Deater-Deckard, K., Chiu, P., Steinberg, L., \& King-Casas, B. (2016). Risky decision making in a laboratory driving task is associated with health risk behaviors during late adolescence but not adulthood. International Journal of Behavioral Development, 40, 58-63. https://doi.org/10.1177/ 0165025415577825 .

Kipping, R. R., Smith, M., Heron, J., Hickman, M., \& Campbell, R. (2015). Multiple risk behavior in adolescence and socioeconomic status: findings from a UK birth cohort. European Journal of Public Health, 25, 44-49. https://doi.org/10.1093/ eurpub/cku078.

Kline, R. B. (2011). Hypothesis testing. Principles and Practice of Structural Equation Modeling (3rd ed., pp. 189-229). New York, NY: Guilford.

Lansford, J. E., \& Bornstein, M. H. (2011). Parenting attributions and attitudes in diverse cultural contexts: Introduction to the special issue. Parenting: Science and Practice, 11, 87-101. https://doi. org/10.1080/15295192.2011.585552.

Lejuez, C. W., Aklin, W. M., Zvolensky, M. J., \& Pedulla, C. M. (2003). Evaluation of the Balloon Analogue Risk Task (BART) as a predictor of adolescent real-world risk-taking behaviours. Journal of Adolescence, 26, 475-479. https://doi.org/10.1016/ S0140-1971(03)00036-8.

Lejuez, C. W., Read, J. P., Kahler, C. W., Richards, J. B., Ramsey, S. E., \& Stuart, G. L., et al. (2002). Evaluation of a behavioral measure of risk taking: The balloon analogue risk task (BART). Journal of Experimental Psychology: Applied, 8, 75-84. https:// doi.org/10.1037/1076-898X.8.2.75

Lotrean, L. M., Laza, V., Ionut, C., \& de Vries, H. (2010). Assessment of health risk behaviours and their interrelationships among young people from two counties of Romania. Journal of Public Health, 18, 403-411. https://doi.org/10.1007/s10389-010-0317-y.

Maticka-Tyndale, E., \& Tenkorang, E. Y. (2010). A multi-level model of condom use among male and female upper primary school students in Nyanza, Kenya. Social Science \& Medicine, 71, 616-625. https://doi.org/10.1016/j.socscimed.2010.03.049.

Mauseth, K. B., Skalisky, J., Clark, N. E., \& Kaffer, R. (2016). Substance use in Muslim culture: Social and generational changes in acceptance and practice in Jordan. Journal of Religion and Health, 55, 1312-1325. https://doi.org/10.1007/s10943-0150064-z.

Maxwell, J. A. (1996). Qualitative research design: An interactive approach. New York, NY: Sage.

Mitchell, S. H., Schoel, C., \& Stevens, A. A. (2008). Mechanisms underlying heightened risk taking in adolescents as compared with adults. Psychonomic Bulletin \& Review, 15, 272-277. https://doi.org/10.3758/PBR.15.2.272.

Osgood, D. W., \& Anderson, A. (2004). Unstructured socializing and rates of delinquency. Criminology, 42, 519-549. https://doi.org/ 10.1111/j.1745-9125.2004.tb00528.x.

Piquero, A. R., Farrington, D. P., \& Blumstein, A. (2003). The criminal career paradigm. In M. Tonry (Ed.), Crime and justice: A review of research (Vol 30, pp. 359-506). Chicago: University of Chicago Press.

Plant, M. A., Plant, M. L., Miller, P., Gmel, G., \& Kuntsche, S. (2009). The social consequences of binge drinking: A comparison of young adults in six European countries. Journal of Addictive Diseases, 28, 294-308. https://doi.org/10.1080/10550880903182978.

Psychological Corporation. (1999). Wechsler abbreviated scale of intelligence. San Antonio, TX: Pearson.

Rahbari, L. (2016). Sexuality in Iran. In C. L. Shehan (Ed.), The Wiley Blackwell Encyclopedia of Family Studies (Vol. 4, pp. 1768-1771), Chichester, West Sussex: John Wiley \& Sons.
Shulman, E. P., Smith, A. R., Silva, K., Icenogle, G., Duell, N., Chein, J., \& Steinberg, L. (2016). The dual systems model: Review, reappraisal, and reaffirmation. Developmental Cognitive Neuroscience, 17, 103-117. https://doi.org/10.1016/j.dcn.2015.12.010.

Smith, A. R., Chein, J., \& Steinberg, L. (2013). Impact of socioemotional context, brain development, and pubertal maturation on adolescent risk-taking. Hormones and Behavior, 64, 323-332. https://doi.org/10.1016/j.yhbeh.2013.03.006.

Spear, L. P. (2013). Adolescent neurodevelopment. Journal of Adolescent Health, 52(Suppl. 2), S7-S13. https://doi.org/10.1016/j.ja dohealth.2012.05.006.

Steinberg, L. (2008). A social neuroscience perspective on adolescent risk-taking. Developmental Review, 28, 78-106. https://doi.org/ 10.1016/j.dr.2007.08.002.

Steinberg, L. (2014). Age of Opportunity: Lessons From the New Science of Adolescence. New York: Houghton Mifflin Harcourt.

Steinberg, L. Albert, D., Cauffman, E., Banich, M., Graham, S., \& Woolard, J. (2008) Age differences in sensation seeking and impulsivity as indexed by behavior and self-report: Evidence for a dual systems model. Developmental Psychology, 44(6), 1764-1778.

Steinberg, L., Icenogle, G., Shulman, E., Breiner, K., Chein, J., \& Bacchini, D., et al. (2017). Around the world, adolescence is a time of heightened sensation seeking and immature selfregulation. Developmental Science. https://doi.org/10.1111/desc. 12532. Advance online publication.

Stevenson, H. W., \& Zusho, A. (2002). Adolescence in China and Japan: Adapting to a changing environment. In B. B. Brown, R. W. Larson, \& T. S. Saraswathi, The World's Youth: Adolescence in Eight Regions of the Globe, (pp. 141-170). New York, NY: Cambridge University Press.

Takakura, M., Nagayama, T., Sakihara, S., \& Willcox, C. (2001). Patterns of health-risk behavior among Japanese high school students. Journal of School Health, 71, 23-29. https://doi.org/10. 1111/j.1746-1561.2001.tb06484.x.

United Nations Development Programme. (2014). Human development report. http://hdr.undp.org/en/data.

World Health Organization. (2014). Adolescents' health-related behaviors. http://apps.who.int/adolescent/second-decade/ section4. Accessed 1 October 2016

Willoughby, T., Good, M., Adachi, P. J. C., Hamza, C., \& Tavernier, R. (2013). Examining the link between adolescent brain development and risk taking from a social-developmental perspective. Brain and Cognition, 83, 315-323. https://doi.org/10.1016/j.ba ndc.2013.09.008.

Xing, Y., Ji, C., \& Zhang, L. (2006). Relationship of binge drinking and other health-compromising behaviors among urban adolescents in China. Journal of Adolescent Health, 39, 495-500. https://doi.org/10.1016/j.jadohealth.2006.03.014.

Natasha Duell is a doctoral candidate in the Department of Psychology at Temple University. Her research explores various mechanisms underling adolescent decision making, particularly with respect to both positive and negative forms of risk taking.

Laurence Steinberg is a Distinguished University Professor and the Laura H. Carnell Professor of Psychology at Temple University. Dr. Steinberg's research has focused on a range of topics in the study of contemporary adolescence, including adolescent brain development, risk-taking and decision-making, parent-adolescent relationships, adolescent employment, high school reform, and juvenile justice.

Grace Icenogle is a doctoral student in the Department of Psychology at Temple University. Her research focuses on adolescent and young 
adult decision making and risk taking as well as the intersection of psychological development, public policy, and the law.

Jason Chein is an Associate Professor in the Department of Psychology at Temple University. Broadly, Dr. Chein's research employs a cognitive neuroscientific approach to evaluate alternative theoretical claims surrounding the basic mechanisms of cognition, the relationship among these mechanisms, and the contribution each makes to high-level cognitive function.

Nandita Chaudhary teaches at the Department of Human Development and Childhood Studies, Lady Irwin College, University of Delhi. She has participated in international collaborations in the area of culture, children's development, and family studies.

Laura Di Giunta is an assistant professor in the Department of Psychology at the Sapienza University of Rome. Her research focuses on individual and cross-cultural differences that predict youths' psychological (mal)adjustment, accounting for socialization factors, especially in regard to emotion regulation-related constructs and social competence.

Kenneth A. Dodge is the William McDougall Professor of Public Policy, Professor of Psychology and Neuroscience, and director of the Center for Child and Family Policy at Duke University. Dodge's research is directed toward understanding how problem behaviors such as delinquency, substance use, school dropout, and child abuse develop across the lifespan, how programs can be developed to prevent these problems, and how public policy can be shaped to improve the public health of communities.

Kostas A. Fanti is an assistant professor in the Department of Psychology at the University of Cyprus. His research explores developmental psychopathology and transactional-ecological models of development, adolescent risk and resilience, and the development and co-occurrence of various externalizing and internalizing behaviors.

Jennifer E. Lansford is a research professor in the Center for Child and Family Policy at Duke University. Her research focuses on the development of aggression and other behavior problems in youth, with an emphasis on how family and peer contexts contribute to or protect against these outcomes. She examines how experiences with parents and peers affect the development of children's behavior problems, and how cultural contexts moderate links between parenting and children's adjustment.

Paul Oburu is a professor in the Department of Educational Psychology at Maseno University. His research focuses on caregiver stress and mental health, children taking care of other siblings, vulnerability factors related to HIV/AIDS infections among adolescents, and risk behaviors amongst this group.

Concetta Pastorelli is a professor in the Department of Psychology at the Sapienza University of Rome. Dr. Pastorelli's main research interests include individual differences during the entire life-span with specific attention to self-efficacy beliefs in the domain of emotional development and interpersonal relationships in adolescence, aggressive behaviors, parenting, and prosocial behavior.

Ann T. Skinner is the research project manager at the Center for Child and Family Policy at Duke University. Her primary research interests include the effects of exposure to violence on children's development, exploring how school-based interventions can be used to improve child academic and behavioral outcomes, and the role that culture and acculturation play in parenting practices and child behavior.

Emma Sorbring is a professor of Child and Youth Studies in the Department of Psychology at University West. Her research and teaching interests lie in the area of children, adolescents and families. Her projects focus on teenagers' internet use and parental strategies, sexual development in traditional and new settings (internet), dating violence, parental behavior and children's adjustment, and young people's decision-making.

Sombat Tapanya is an assistant professor in the Department of Psychiatry at Chiang Mai University. Dr. Tapanya's research interest is in the area of violence prevention in children and youths.

Liliana Maria Uribe Tirado is a research professor at Universidad San Buenaventura. Her main research focuses on parenting and child adjustment in different cultural contexts. She is also actively involved in testing evidence based prevention programs.

Liane Peña Alampay is an Associate professor in the Department of Psychology at the Ateneo de Manila University. Her research is in the areas of Filipino parenting and its relation to child and adolescent development, cultural aspects of parenting and family relationships, and interventions for children and families at risk and in difficult circumstances.

Suha M. Al-Hassan is an associate professor of early childhood and special education at the Emirates College for Advanced Education. Dr. Al-Hassan's research focuses on parenting programs, school readiness, children's behavior, and inclusion.

Hanan M. S. Takash is an assistant professor at the Queen Rania Faculty for Childhood at Hashemite University. Her research focuses on multicultural parenting practices and child behavioral outcomes.

Dario Bacchini is an association professor of developmental psychology at the University of Campania "Luigi Vanvitelli". His research interests include moral development, bullying at school, antisocial and prosocial behavior, parental disciplinary styles, and effects of exposure to violent environments on child and adolescent adjustment.

Lei Chang is the chair professor and head of the Department of Psychology at the University of Macau. His research interests include child development, evolutionary psychology, and applied measurement and statistics. 